Florida International University FIU Digital Commons

\title{
Anxiety Sensitivity's Facets in Relation to Anxious and Depressive Symptoms in Youth
}

Kristin A. Nichols-Lopez

Florida International University, knichols225@aol.com

DOI: $10.25148 /$ etd.FI10081213

Follow this and additional works at: https://digitalcommons.fiu.edu/etd

\section{Recommended Citation}

Nichols-Lopez, Kristin A., "Anxiety Sensitivity's Facets in Relation to Anxious and Depressive Symptoms in Youth" (2010). FIU Electronic Theses and Dissertations. 268.

https://digitalcommons.fiu.edu/etd/268 


\section{FLORIDA INTERNATIONAL UNIVERSITY}

Miami, Florida

\section{ANXIETY SENSITIVITY'S FACETS IN RELATION TO}

ANXIOUS AND DEPRESSIVE SYMPTOMS IN YOUTH

A dissertation submitted in partial fulfillment of the

requirements for the degree of

DOCTOR OF PHILOSOPHY

in

PSYCHOLOGY

by

Kristin Nichols-Lopez

2010 


\section{To: Dean Kenneth Furton}

College of Arts and Sciences

This dissertation, written by Kristin Nichols-Lopez, and entitled Anxiety Sensitivity's Facets in Relation to Anxious and Depressive Symptoms in Youth, having been approved in respect to style and intellectual content, is referred to you for judgment.

We have read this dissertation and recommend that it be approved.

William Kurtines

Paulette Johnson

James Jaccard, Co-Major Professor

Wendy Silverman, Co-Major Professor

Date of Defense: July 15, 2010

The dissertation of Kristin Nichols-Lopez is approved.

\begin{tabular}{r}
$\begin{array}{r}\text { Dean Kenneth Furton } \\
\text { College of Arts and Sciences }\end{array}$ \\
\hline Interim Dean Kevin O'Shea \\
University Graduate School
\end{tabular}

Florida International University, 2010 


\section{DEDICATION}

I dedicate this thesis to my entire family, and especially to my mother, Angela, who taught me the value of strength and the power of perseverance. Without my family's faith, patience, support, and love, the completion of this work would not have been possible. 


\section{ACKNOWLEDGMENTS}

I owe a great deal of thanks to many people for making this dissertation possible. First, I would like to thank the members of my committee, Dr. William Kurtines, Dr. Paulette Johnson, and especially my major professors, Dr. Wendy Silverman and Dr. James Jaccard. It was all of their guidance, support, patience, and advice that enabled me to navigate through my entire graduate student career.

I am especially grateful to Dr. Wendy Silverman for her years of mentoring. I would not be where I am today without her insight, sincerity, and understanding. I don't know if there are words to describe how much I have learned from her. I thank Dr. James Jaccard for giving his support throughout the years. My work would not have been possible without his assistance and statistical expertise. The knowledge I gained working for Dr. Paulette Johnson gave me the foundation I needed to excel. I am thankful for my supervisor, Dr. Jerome Levitt, for our many discussions, and for his continual enthusiasm and support of my pursuit of a Ph.D.

I also wish to thank Carla Marin and Yasmin Rey for being exceptionally great and loving friends who were always there for me when I needed them most. None of this would have been as enjoyable if I had not had them standing by me each step of the way. And finally, to my family, your encouragement and love carried me through this. You always believed in me and provided me with the means to see this through. I never could have imagined that one of the hardest parts of writing my dissertation would have been finding the right words to say thank you. My words fall short of the gratitude I feel. 


\section{ABSTRACT OF THE DISSERTATION \\ ANXIETY SENSITIVITY'S FACETS IN RELATION TO \\ ANXIOUS AND DEPRESSIVE SYMPTOMS IN YOUTH}

by

Kristin Nichols-Lopez

Florida International University, 2010

Miami, Florida

Professor James Jaccard, Co-Major Professor

Professor Wendy Silverman, Co-Major Professor

Anxiety sensitivity is a multifaceted cognitive risk factor currently being examined in relation to anxiety and depression. The paucity of research on the relative contribution of the facets of anxiety sensitivity to anxiety and depression, coupled with variations in existing findings, indicate that the relations remain inadequately understood. In the present study, the relations between the facets of anxiety sensitivity, anxiety, and depression were examined in 730 Hispanic-Latino and European-American youth referred to an anxiety specialty clinic. Youth completed the Childhood Anxiety Sensitivity Index, the Revised Children's Manifest Anxiety Scale, and the Children's Depression Inventory. The factor structure of the Childhood Anxiety Sensitivity Index was examined using ordered-categorical confirmatory factor analytic techniques. Goodness-of-fit criteria indicated that a two-factor model fit the data best. The identified facets of anxiety sensitivity included Physical/Mental Concerns and Social Concerns. Support was also found for cross-ethnic equivalence of the two-factor model across Hispanic-Latino and European-American youth. Structural equation modeling was used 
to examine models involving anxiety sensitivity, anxiety, and depression. Results indicated that an overall measure of anxiety sensitivity was positively associated with both anxiety and depression, while the facets of anxiety sensitivity showed differential relations to anxiety and depression symptoms. Both facets of anxiety sensitivity were related to overall anxiety and its symptom dimensions, with the exception being that Social Concerns was not related to physiological anxiety symptoms. Physical/Mental Concerns were strongly associated with overall depression and with all depression symptom dimensions. Social Concerns was not significantly associated with depression or its symptom dimensions. These findings highlight that anxiety sensitivity's relations to youth psychiatric symptoms are complex. Results suggest that focusing on anxiety sensitivity's facets is important to fully understand its role in psychopathology. Clinicians may want to target all facets of anxiety sensitivity when treating anxious youth. However, in the context of depression, it might be sufficient for clinicians to target Physical/Mental Incapacitation Concerns. 


\section{TABLE OF CONTENTS}

CHAPTER

PAGE

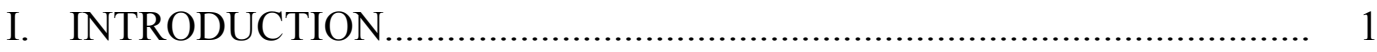

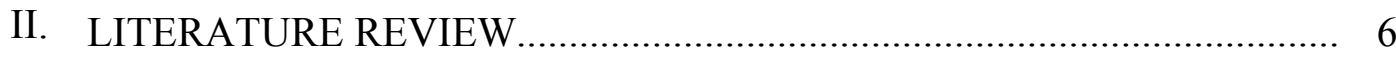

Psychometric Assessment of AS in Youth.................................................... 8

Research on AS with Youth Samples..................................................... 18

Meta-analyses with AS................................................................. 29

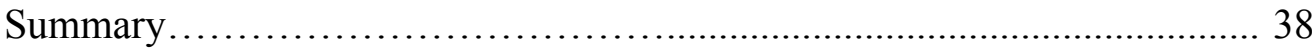

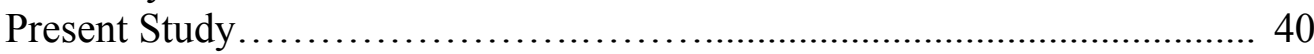

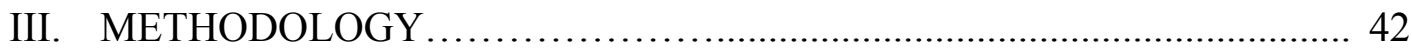

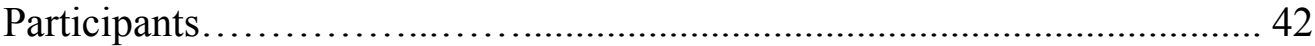

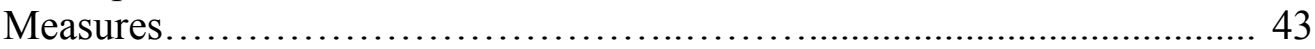

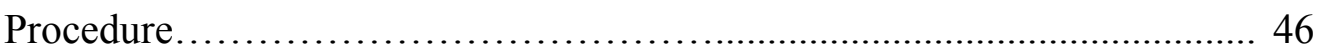

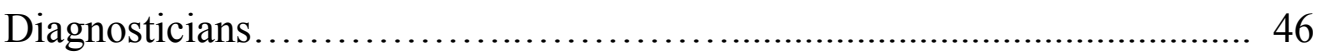

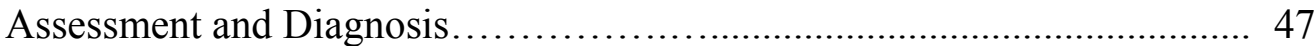

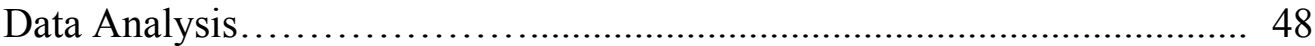

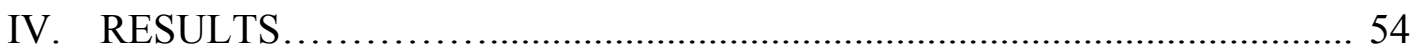

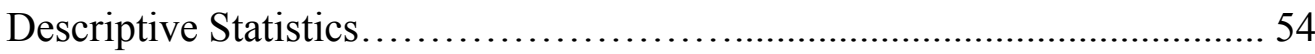

Factor Structure of the CASI........................................................... 54

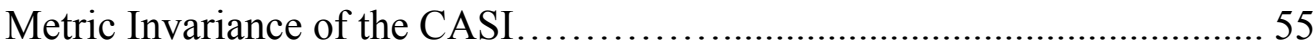

AS, Anxiety, and Depression.................................................................. 57

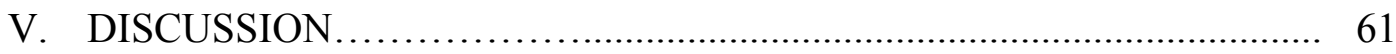

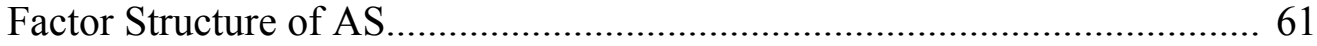

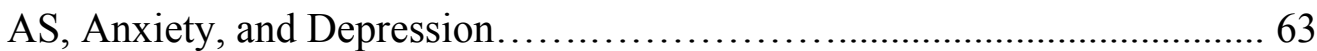

Limitations and Future Directions........................................................ 66

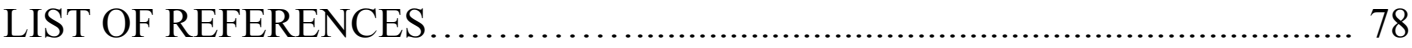

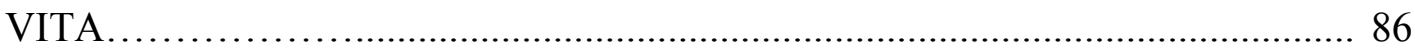




\section{LIST OF TABLES}

TABLE

PAGE

1. Factor Analytic Studies of the Childhood Anxiety Sensitivity Index............. 70

2. Socio-demographic Characteristics of Participants.......................... 71

3. Means, Standard Deviations, and Relevant Estimates for all Measures........... 71

4. Goodness-of-fit Indices for 18-item Version of CASI........................ 72

5. Standardized Factor Loadings for the Two-Factor CASI Model................. 72

6. Standardized Threshold Estimates for the Two-Factor CASI Model............. 73 


\section{CHAPTER I}

\section{INTRODUCTION}

Current developmental models of anxiety emphasize not only the etiologic role of biological factors in children and adolescents, but also cognitive vulnerabilities in the development and maintenance of anxiety disorders (e.g., Barlow, 2002; Taylor, 1995; Vasey \& Dadds, 2001). Anxiety sensitivity (AS), a cognitive construct that has received growing research attention in recent years (Naragon-Gainey, 2010; Olatjuni \& WolitzkyTaylor, 2009; Silverman \& Weems, 1999), refers to the belief that an individual's anxious symptoms may lead to negative consequences that can be physical, psychological, or social in nature (Reiss, 1991). Initially, AS was viewed as a specific risk factor for anxiety and its disorders (Reiss \& McNally, 1985; Reiss, Peterson, Gursky, \& McNally, 1986). More recent research suggests a relation between AS and depression (Joiner, et al., 2002; Lambert, McCreary, Preston, Schmidt, Joiner, \& Ialongo, 2004; Naragon-Gainey, 2010; Weems, Hammond-Laurence, Silverman, \& Ferguson, 1997).

The relations between AS and anxiety, as well as between AS and depression, are likely to be complex. Research confirms that AS is multidimensional (Silverman, Goedhart, Barret, \& Turner, 2003). Some studies have found support for a two-factor model (Chorpita \& Daleiden, 2000; Lambert, Cooley, Campbell, Benoit, \& Stansbury, 2004), with each study reporting different a two-factor structure of the CASI. Other studies have supported a three-factor model (Essau, Sasagawa, \& Ollendick, 2010; Muris, Schmidt, Merckelbach, \& Schouten, 2001; Silverman et al., 2003). The three-factor model of AS include Physical Concerns (e.g., When my stomach hurts, I worry that I might be really sick), Mental Incapacitation Concerns (e.g., When I am afraid, I worry 
that I might be crazy), and Social Concerns (e.g., I don't want others to know I'm afraid). The strongest support has been found for a four-factor model (Adornetto, Hensdiek, Meyer, In-Albon, \& Schneider, 2008; Muris et al., 2001; Silverman et al., 2003) (see Table 1 for a summary). The four-factor model is similar to the three-factor model, with the only difference being that the third factor of Physical Concerns is broken down into Disease Concerns (e.g., It scares me when my heart beats fast) and Unsteady Concerns (e.g., It scares me when I feel faint).

Viewing AS as a multidimensional construct has led investigators to highlight the importance of determining whether, and how, AS's specific facets are differentially related to psychopathology, particularly to internalizing disorders such as anxiety and depression (see Chapter 2, Literature Review for further details). Although research has consistently found a relationship between the different facets of AS and anxiety (Kearney, Albano, Eisen, Allan, \& Barlow, 1997; Weems, Hayward, Killen, \& Taylor, 2002), results have been mixed with respect to the relations between AS and depression. Some studies have found support for differential relations between the facets of AS and depression (Lambert, McCreary, Preston, Schmidt, Joiner, \& Ialongo, 2004); others have found no relationship at all between AS and depression (Dia \& Bradshaw, 2008; Joiner, et al., 2002).

Joiner et al. (2002) conducted the first study that examined the relations between the facets of AS and anxiety and depression with a sample of youth psychiatric inpatients. Using two of the AS factors, Physical Concerns and Mental Incapacitation Concerns, Joiner et al. found that both the Physical Concerns and Mental Incapacitation Concerns facets of AS were significantly associated with anxiety after controlling for depression. 
Joiner et al. also reported that both Physical Concerns and Mental Incapacitation Concerns were not associated with depression after controlling for anxiety. Their results were contrary to their initial hypothesis based on the adult literature (Schmidt, Lerew, \& Joiner, 1998), that Mental Incapacitation Concerns would predict depression scores.

Lambert, McCreary et al. (2004) also examined the relations between the facets of AS, anxiety, and depression in a community sample of urban African-American adolescents. Unlike the findings of Joiner et al., Lambert et al. found a significant relation between AS and depression, with Mental Incapacitation Concerns having a positive association with depression, and Fear of Cardiovascular sensations having a negative association with depression. In the most recent study to examine the issue, Dia and Bradshaw (2008) reported that among youth with internalizing disorders (i.e., diagnoses of anxiety and depressive disorders) Mental Incapacitation Concerns were not significantly related to depression, after controlling for negative affect.

Taken together, the results from these studies indicate that the AS-depression association in youth remains ambiguous. The paucity of research on the relative contribution of the facets of AS to anxiety and depression, coupled with variations in existing findings, led to the present study. Theoretically, clarification of the relative contributions of the facets of AS to anxiety and depression is important to further develop cognitive-based models of these psychiatric conditions. Clinically, examining the unique contribution of each of the facets of AS to anxiety and depression may help to identify the specific types of cognitions (e.g., Mental Incapacitation Concerns) that may be targeted when conducting cognitive behavioral therapy to reduce anxiety and/or depression in youth. 
The main purpose of the present dissertation study was to clarify the relations between the facets of AS with anxiety and depression in a large sample of HispanicLatino and European-American youth referred to an anxiety disorders specialty clinic. Because questions remain as to the exact composition of AS factors, this study also formally examined the factor structure of AS using the Childhood Anxiety Sensitivity Index (CASI; Silverman, Fleisig, Rabian, \& Peterson, 1991) to determine which of the proposed structures found in past studies provided the best fit for the current sample.

The measurement equivalence of best-fitting factor model of the CASI was examined across Hispanic-Latino and European-American youth. Evidence for measurement equivalence of the instrument used to assess AS (i.e., the CASI) is necessary in order to use the measure appropriately across groups. If measurement equivalence is not established, it is possible that differences or similarities in AS among groups may simply reflect measurement bias rather than true findings. If measurement equivalence is established and different groups are responding to the AS items in the same way, it can be inferred that members of these groups are interpreting AS and its facets similarly (Vandenberg \& Lance, 2000). To date, no study has examined the measurement equivalence of the CASI across Hispanic-Latino and European-American youth.

This dissertation is divided into the following chapters. An overview of the research that documents the etiology of AS a distinct, multidimensional construct is presented first. This is followed by subsequent research relating AS to internalizing disorders in youth. The paucity of research on the relation of the facets of AS to anxiety and depression in youth is also discussed. Recent meta-analytic studies summarizing a 
large body of literature involving the relations between the facets of AS, anxiety, and depression with adult samples then follows (Chapter 2). Upon review of this literature, it became evident that issues regarding the understanding of AS as it relates to anxious and depressive symptoms in youth required additional attention. These issues set the stage for the methodology employed and the specific research questions examined in this study. The next section (Chapter 3) discusses the methodology used to address the posed research questions. This is followed by a presentation of the Dissertation findings (Chapter 4). Implications for researchers and clinicians, study limitations and future directions for research are summarized in the final section (Chapter 5). 


\section{CHAPTER II}

\section{LITERATURE REVIEW}

A cognitive construct receiving increased attention recently (see Silverman \& Weems, 1999; Olatjuni \& Wolitzky-Taylor, 2009; Naragon-Gainey, 2010), AS refers to the belief that one's anxious symptoms may lead to negative physical, psychological, or social consequences (Reiss \& McNally, 1985; Reiss, Peterson, Gursky, \& McNally, 1986). The original concept of AS was formed within the framework of Reiss' expectancy theory (1991), which states that a set of fundamental fears, including AS, serve to amplify fear, anxiety, and panic. These fundamental fears are thought to be distinct from common fears (e.g., animal fears, situational fears) for two reasons: (1) fundamental fears are naturally aversive to the majority of people, and (2) fundamental fears are logical reductions of common fears. Differences in AS levels could explain individual differences in the conditioning of fear and consequences related to fear, and AS was considered an "amplication factor" (Taylor \& Fedoroff, 1999) ${ }^{1}$. These individual differences are believed to result from variations in the genes that make anxiety an annoyance and the influence of cognitive factors that lead to the formation of beliefs that anxiety symptoms will lead to negative consequences (Reiss, Silverman, \& Weems, 2001).

Initially, AS was considered a specific risk factor for panic disorder, as panic attacks were thought to result from "catastrophic misinterpretations" of bodily reactions

\footnotetext{
${ }^{1}$ It is important to note that empirical evidence exists in contradiction of expectancy theory (see Taylor \& Fedoroff, 1999). Although there is sufficient evidence that AS is indeed a relevant construct to the development of psychopathology, other theories may provide better explanations as to why.
} 
(Clark, 1986; Reiss, 1987). For example, one may interpret a rapid heartbeat as catastrophic and presume this symptom to be a precursor to a heart attack. Such an interpretation would initiate a positive feedback loop through which the negative interpretation of physical symptoms would only serve to intensify the symptoms themselves, resulting in a panic attack. This model suggests that people who suffer from panic attacks would be more likely to make cognitive misinterpretations of physical symptoms (i.e., have elevated AS). Numerous studies have been conducted, initially with adult samples (Cox, Parker, \& Swinson, 1996; Taylor, Koch and McNally, 1992) and later with youth samples (Hayward, Killen, Kraemer, \& Taylor, 2000; Kearney, Albano, Eisen, Allan \& Barlow, 1997), to support the notion that AS is significant predictor of panic. Subsequent studies have shown elevated levels of AS in adults with various types of anxiety disorders (Olatjuni \& Wolitzky-Taylor, 2009; Naragon-Gainey, 2010; Taylor, Koch, \& Mcnally, 1992).

Early research on the concept of AS was also concerned with whether AS was conceptually distinct from trait anxiety (Marin, Rey, Nichols-Lopez, \& Silverman, 2008). Lilienfeld, Jacob, and Turner (1989) argued for the possibility that the questionnaire developed to measure AS was simply measuring trait anxiety, or the tendency to respond with fear to stressors. Key differences between AS and trait anxiety have been explained in the literature (McNally, 1996; Reiss, 1997). Trait anxiety predicts a proneness to respond anxiously to aversive stimuli, which is regarded as dangerous, while AS predicts proneness to respond anxiously to the specific symptoms of anxiety. In AS, however, the symptoms themselves are not the feared stimuli: It is the fact that they may be uncontrollable that makes the symptoms seem scary. 
Subsequent research has empirically demonstrated that AS is indeed distinct from trait anxiety in both adult (Marian \& McNally, 1996; McNally, 1989) and youth samples (Weems, Hammond-Laurence, Silverman, \& Ginsburg, 1998). These findings allowed researchers to move on to more pointed questions contemplating the relations of AS with various types of psychopathology. Accordingly, research has indicated that AS is related to anxiety and is also linked with depression (Otto, Pollack, Fava, Ucello, \& Rosenbaum, 1995; Taylor, Koch, Woody, \& McLean, 1996), chronic pain (see Asmundson, 1999), substance abuse (see Stewart, Samoluk, \& MacDonald, 1999); and is elevated in clinical samples compared to normal control samples (see Taylor, 1999). Psychometric Assessment of AS in Youth

As with most research on theories concerning etiology of psychiatric disorders, research on AS involving adults preceded research on children (Silverman \& Weems, 1999). To bridge the gap in the extant literature and adequately examine AS in youth, Silverman, Fleisig, Rabian, and Peterson (1991) developed the Childhood Anxiety Sensitivity Index (CASI), a "downward extension" of the most common measure of AS used in adults, the Anxiety Sensitivity Index (ASI; Peterson \& Reiss, 1987). The CASI is a self-report questionnaire consisting of 18 items designed to assess the extent to which youth view the experience of anxiety-related symptoms as aversive (e.g., It scares me when I feel shaky). Respondents rate their agreement to each item along a scale of 1 (None) to 3 (A lot). Total scores range from 18 to 54, with higher scores indicating higher levels of AS.

In the initial study, the CASI was administered to both non-clinic $(n=72 ; M=$ 13.3 years) and clinic ( $n=33 ; M=10.6$ years) samples of youth. Results indicated an 
internal consistency $(\alpha)$ coefficient of .87 for the total score with both samples; and, testretest correlations of .79 and .76 for the non-clinic and clinic samples, respectively. In addition, after controlling for anxiety, the CASI accounted for $48 \%$ and $35 \%$ of additional variance in the prediction of fear as measured by the Fear Survey Schedule for Children-Revised (FSSC-R; Ollendick, 1983) with the non-clinic and clinic samples, respectively.

Factor Structure. As with adult research (Olatjuni \& Wolitzky-Taylor, 2009), current views of the construct of AS in children have been heavily influenced by psychometric research involving the factor structure of the CASI. Although Reiss \& McNally (1985) initially conceptualized AS as uni-dimensional, several studies with youth involving the CASI have reported evidence that AS consists of more than one factor. The continued refinement of AS theory involving the number and item-structure of the facets would improve research on AS, especially if the different facets demonstrate specificity with regard to different psychological constructs (Silverman et al., 2003).

Examination of Table 1 indicates that studies have found support for a twofactor model (Chorpita \& Daleiden, 2000; Lambert, Cooley, Campbell, Benoit, \& Stansbury, 2004), a three-factor model (Essau, Sasagawa, \& Ollendick, 2010; Muris, Schmidt, Merckelbach, \& Schouten, 2001; Silverman et al., 2003), and a four-factor model (Adornetto, Hensdiek, Meyer, In-Albon, \& Schneider, 2008; Muris et al., 2001; Silverman et al., 2003), with some studies finding support for more than one of the above models. In the subsequent paragraphs, the most important studies involving the examination of the factor structure of the CASI are reported in detail. 
Silverman, Ginsburg, and Goedhart (1999) conducted the first factor analytic study on the CASI, using both a clinic ( $n=258$; ages 7 to 16 years, $M=10.4$ years $)$ and non-clinic sample ( $n=249$; ages 7 to 12 years, $M=9.9$ years) of youth. Hierarchical models were developed, using data from the clinic sample, by performing four separate EFA models with two methods of extraction, principal components analysis (PCA) and principal axis factoring (PAF); and two methods of rotation, oblimin and varimax. Models involving one higher-order factor, with two, three or four first-order factors, were identified. Subsequently, a variety of fit statistics for the various CFA models were evaluated, using data from the non-clinic sample to test for model fit. The performance of the viable models against one another was evaluated with chi-square difference tests. The model with four first-order factors fit the data best, though the model with three firstorder factors also fit the data well. In addition, the second-order factors accounted for more than $50 \%$ of the explained variance in the CASI. The four factors were labeled as follows: (1) Physical Concerns, (2) Mental Incapacitation Concerns, (3) Social Concerns, and (4) Control. The study's findings supported the multidimensional nature of AS in youth, while also providing validity for the usefulness of the overall CASI score.

In a subsequent factor analysis involving the CASI, Chorpita and Daleiden (2000) tested models on a clinic sample of children and adolescents with anxiety disorders, ages 7 to 17 years ( $N=228 ; M=12$ years, 9 months; $S D=2$ years, 9 months). To evaluate the factor structure, EFAs were conducted using both PCA and PAF extraction methods. Both EFAs revealed a one-factor solution. The items with the strongest factor loadings were, for the most part, "autonomic" in nature. Subsequently, the authors conducted CFAs using maximum likelihood (ML) estimation procedures, 
testing one- and two-factor models separately in both children (ages 7 to 11) and adolescents (ages 12 to 17). The two-factor model consisted of autonomic and nonautonomic factors, with the items having been classed into groups by independent raters. A variety of fit indices were evaluated to test model fit. Chi-square difference tests were computed to test competing models. For both children and adolescents, the two-factor model fit the data best. However for the models tested, the Root Mean Square Error of Approximation (RMSEA) estimates, measures of model fit with a penalty for lack of parsimony, were .12 and .10 , for children and adolescents, respectively. These estimates are both above the cutoff suggested for reasonable model fit, .08 (Hu \& Bentler, 1999), suggesting that neither model provided a good fit for the data.

Next, Muris, Schmidt, Merckelbach, and Schouten (2001) examined a 16-item version of the CASI in a large sample of non-clinic Dutch adolescents, ages 13 to 16 years $(N=819 ; M=14.2$ years; $S D=1.0)$. Only the items on the CASI that directly corresponded to the 16 items on the ASI were included, and CFAs were conducted using ML estimation procedures. Ten competing models were examined, consisting of one, two, three, or four lower-order AS factors. Some of the factor structures tested also modeled a higher-order factor of AS. A variety of fit indices were evaluated for all models; however, chi-square significance tests between nested models were not performed. Results indicated that both a three-factor model with one higher-order factor and a four-factor model with one higher-order factor fit the data best. These results are similar to those found by Silverman et al. (1999), indicating that both a 3- and 4-factor model, each having a higher-order factor, fit the data well. 
With a debate over the factor structure of the CASI still present in the literature, Silverman, Goedhart, Barret, and Turner (2003) examined the goodness-of-fit of models reported in literature in order to select the "best model in terms of fit." Evidence at that time indicated that the CASI was comprised of a higher-order AS factor, but the exact number and composition of the lower-order factors was still in question. Data were collected from a non-clinic sample of Australian youth, ages 7 to 15 years $(n=$ 767; $M=12.2$ years, $S D=1.9)$. These data were combined with both samples used in Silverman et al. (1999). First, CFAs were conducted using ML estimation to select the best model from 13 items on the CASI that consistently loaded on lower-order factors, based on a review of the available literature. Models with one factor, two correlated factors, three correlated factors and four correlated factors were tested. A variety of fit indices were evaluated, and chi-square difference tests were used to select the best-fitting model. The four correlated factor model fit the data best.

Next, the five items that were not classed based on inconsistencies in previous studies were added into the models using modification indices with a derivation sample. Using a cross validation sample, CFAs were used to select the best-fitting model, using all 18 CASI items. Again, the four correlated factor model fit the data best; though overall model fit, as indicated by various fit statistics, decreased when compared with the 13-item version. Metric invariance of the 18-item, four-factor model was examined across age (children: 7 to 11 years, adolescents: 12 to 17 years) and youth sex using the Australian cross-validation sample by constraining the factor loading across groups. In both cases, model fit did not degrade when model constraints were imposed, indicating that factor loadings are operating similarly across both age and youth sex. The internal 
consistencies of the four factor scales ranged from moderate to good ( $\alpha=.62$ to .80$)$.

The four factors were labeled as follows: (1) Disease Concerns, (2) Unsteady Concerns, (3) Mental Incapacitation Concerns, and (4) Social Concerns, after labels proposed by Carter, Miller, Sbrocco, Suchday, and Lewis (1999) in a factor analysis of the ASI conducted with a sample of African-American college students. Among the factors, the strongest association was found between Disease Concerns and Unsteady Concerns, $r=.68$; the lowest association between Mental Incapacitation Concerns and Social Concerns, $r=.21$; with the remaining associations being between .36 and .58 . Finally, a higher-order model was examined; and again, a single higher-order factor was found which accounted for a substantial gain in explained variance.

Using data from a community sample of inner-city African-American youth, ages 8 to $12(N=144 ; M=10.32)$, Lambert, Cooley et al. (2004) evaluated the factor structure of the CASI as reported by Silverman et al. (1999) by conducting CFAs using ML estimation. Fit indices were examined for three- and four-factor hierarchical models. Both models provided better fit than a single-factor model and the RMSEA estimates were in the acceptable range. However, the Comparative Fit Index (CFI) and the Tucker Lewis Index (TLI) values were below the cutoff suggested for reasonable model fit, .90 (Hu \& Bentler, 1999), suggesting that the model did not provide a good fit for the data. Though, CFI and TLI are among the fit indices least affected by sample size (Fan, Thompson, \& Wang, 1999), it is still possible that a sample of 144 youths was not large enough to evaluate the model, given the size of the observed covariance matrix used in the analysis. As a result of poor model fit, Lambert, Cooley et al. conducted EFAs using both PAF and PCA extraction with oblique rotation to determine a factor structure that fit 
with their data. A solution with six initial factors emerged, but was reduced to two factors, Physical Concerns (consisting of six items) and Mental Incapacitation Concerns (consisting of five items) because of conceptual incongruities.

The two most recent factor analytic studies on the CASI (Adornetto et al., 2008; Essau et al., 2010) have been conducted using German samples. Adornetto et al. tested both the 13-item and 18-item models proposed by Silverman et al. (2003) on four samples consisting of children ages 9 to $16,(N=1244,225,230$, and 143) using CFA procedures. The authors do not indicate the estimation procedures used in their CFA. Although the 13-item CASI consistently performed better than the 18-item CASI, the four-factor model performed better on average than the three-factor model within both versions, though no chi-square difference tests were conducted. It should be noted that with the smaller samples, some of the fit indices were not in the satisfactory range. In addition, the authors performed their own EFA (using data from sample 1) using both varimax and oblimin rotations, which also resulted in a four-factor model using 17 items.

Metric invariance was examined across age (children: 8 to 12 years; adolescents: 12 to 16 years) and youth sex using the 13-item CASI, with four factors, (the model shown to have the best fit). In this case, metric invariance was not supported as model fit degraded when model constraints were imposed, indicating that factor loadings were not operating similarly across both age and youth sex in the German sample. Overall, both the 13- and 18-item, four-factor structure proposed by Silverman et al. (2003) was able to be replicated with a sample of German youth, the results regarding factorial invariance across age and youth sex were not replicated. Adornetto et al. (2008) note that the results regarding factorial invariance should be taken with caution due to 
sampling issues.

In the most recent factor analytic study, Esau et al. (2010) collected data from a non-clinic sample of German youth, ages 12 to 17 years $(N=1,292 ; M=14.6$ years, $S D$ = 1.6). The total sample was split in half, and an EFA was conducted using ML estimation with a promax rotation on the first half of the sample. Then, a CFA was conducted on the models resulting from the EFA with the other half of the sample. The authors did not indicate the estimation procedures used in their CFA. The authors concluded that the three-factor model provided a better fit for the data. However, the Goodness-of-Fit Index (GFI) reported for the three- and four-factor models was .87 and .86 respectively. These estimates are well below the currently suggested .95 cutoff specified for adequate model fit (Schumacker \& Lomax, 2004). As the GFI often has an upward bias with large samples, models with values below .95 may not be considered a good fit for the data. Moreover, the authors only provide fit indices that measure absolute fit, which are based on predicted versus observed covariances. Jaccard and Wan (1996) recommend use of at least three fit tests from three separate classes; (1) indices of absolute fit, (2) indices with a penalty function for lack of parsimony, and (3) indices of comparative fit, which test the specified model against a null model.

Summary. There is still considerable debate in the literature as to which factor structure fits the CASI best. Given the above, further examination of the factor structure of AS is warranted. Continued refinement of the AS construct through additional factor analytic studies would advance research on AS, especially if the facets of AS demonstrate specificity with regard to different psychological constructs (Silverman et al., 2003). In addition, finding evidence for invariance across Hispanic-Latinos and European- 
American youth would validate past research conducted with these groups and provide an empirical basis for conducting future research on AS with these groups.

The disparity in factor structures found to date may be the result of differences in sample characteristics and study methodology. Each of the factor-analytic studies involving the CASI employed different statistical methodologies for analyzing the data. Some studies have used only EFAs or CFAs, while others have used both. Even within EFA and CFA frameworks, the different estimation procedures used can produce different results.

The correlated four-factor model proposed by Silverman et al (2003) seems to have the most promise, as it was developed using both a non-clinic and clinic sample, was revealed to be the best-fitting model over others after using formal statistical comparisons; and factor loadings were found to be proportionally equal across age and youth sex. While the four-factor model proposed by Silverman et al. (2003) was not able to be replicated using data from a community sample of inner-city African-American youth (Lambert, Cooley et al., 2004), it was replicated using data from a community sample of German youth (Adornetto et al., 2008), though variance was found with respect to factor loadings across age and youth sex. The inability to replicate these results may have merely been a function of sample size and composition.

It is still unclear which factor model of the CASI will fit best with minority youth (i.e. African-Americans and Hispanic-Latinos), as the model has only fit well with data from primarily European-American, Dutch and German samples. Limited support for metric invariance, a type of measurement invariance, of the CASI has been found across youth sex and age, and metric invariance has not been examined across ethnic groups. 
Measurement invariance refers to the extent that a measure assesses a construct in the same manner across distinct groups (Hui \& Triandis, 1985). Measurement invariance of the questionnaire being used to assess AS - in this case the CASI - should be determined before using AS across two or more groups (Vandenberg \& Lance, 2000). As such, measurement equivalence research is not something that should be viewed as simply being the purview of assessment researchers or psychometricians, but as a necessary initial step to conducting research with a given questionnaire.

Comparisons made with questionnaires used to assess a latent construct assume theoretical equivalence of latent variables, associational equivalence between items and latent variables, and that items are influenced to the same degree by unique factors not being measured (Vandenberg \& Lance, 2000). Metric invariance is only one piece of the measurement invariance puzzle that can be examined. Various types of measurement invariance have been described in the literature, including configural invariance, metric invariance and invariant uniqueness. The use of terminology describing available statistical techniques associated with measurement invariance has been inconsistent (Vandenberg \& Lance, 2000; Knight \& Zerr, 2010).

For the purposes of this paper, the definitions proposed by Vandenberg and Lance (2000) will be used. Configural invariance refers to the equivalence of a measure's pattern of loadings across groups, without involving constraints. Metric invariance refers to equivalence of the factor loadings of the items comprising each factor across groups. Invariant uniqueness refers to the equivalence of the error terms associated with items across groups. Configural invariance, metric invariance, and invariant uniqueness can be examined using multiple group CFA techniques (Millsap \& Yun-Tein, 2004). To date, 
no study has examined measurement equivalence of the CASI involving different ethnic groups.

Finally, it is important to note that the items on the CASI are ordinal in nature. When conducting item-level factor analysis of such items, categorical variable methodology should be applied in order to avoid problems resulting from the application of traditional continuous factor methodology (Millsap \& Yun-Tein, 2004). Traditional ML CFA techniques as used in all of the CASI CFAs published to date, produce measurement bias, particularly, underestimated parameters and overestimated standard errors, when used with ordered-categorical items (Babakus, Ferguson, \& Joreskog, 1987). Currently, none of the factor-analytic studies on the CASI have employed orderedcategorical modeling to the data.

Research on AS with Youth Samples

Although most of the initial research on AS was conducted with adult samples, the development of the CASI has done much to advance research on AS in youth. Many of the original questions proposed and examined in the adult literature have now been replicated with youth samples.

Trait Anxiety and AS in youth. Even while the issue of AS being distinct from trait anxiety was being answered in the adult literature (McNally, 1989; Marian \& McNally, 1996); questions still remained as to whether AS (as measured by the CASI) and trait anxiety were distinct constructs for youth, particularly for younger children (Chorpita, Albano, \& Barlow, 1996). Chorpita et al. (1996) examined the incremental validity of the CASI with a clinic sample of youth diagnosed with an anxiety disorder ( $N=112$, ages 7 to 17 ). The authors used multiple regressions to examine the ability of 
the CASI to predict trait anxiety scores, as measured by the Trait subscale of the StateTrait Anxiety Inventory for Children (STAIC-T; Spielberger, 1973), and fear scores as measured by the FSSC-R. Using two separate regression models, STAIC-T and FSSC-R scores were regressed onto CASI scores, age, the product term of CASI and age squared, and the Physiological Symptoms subscale (RCMAS-P; Reynolds \& Richmond, 1978) of the Revised Children's Manifest Anxiety Scale (RCMAS). In both models, the authors found the interaction term to be significant indicating that the incremental validity of the CASI increased with age in a curvilinear fashion. The authors then split the sample into two groups, younger ( $n=43$, ages 7 to 11 ) and older ( $n=69$, ages 12 to 17). Hierarchical multiple regression analyses were used to predict STAIC-T scores from CASI scores and either RCMAS-P scores or FSSC-R scores. Results indicated that the CASI only predicted additional variance for the older sample. Though the results support the idea that AS predicts trait anxiety in older youth, the authors state that the CASI may not provide and accurate measure of AS when used with younger children.

Weems et al. (1998) pointed out several difficulties with the conclusions presented by Chorpita et al. (1996). Most importantly, Weems et al. noted that the issue raised by Lilienfeld (1989) in the adult literature was not whether AS predicted trait anxiety, but whether AS predicted additional variance beyond trait anxiety. The methodology employed by Chorpita et al. did not address this question. Also, the small sample sizes used by Chorpita et al. may not have had enough predictive power to detect significant effects. Weems et al. sought to clarify this issue by conducting various multiple regression analyses on a clinic sample of 280 youth (ages 6 to $17, M=10$ years) diagnosed with a primary anxiety disorder. As in the previous study, the sample was split 
into two age groups, younger (ages 6 to 11) and older (ages 12 to 17). Scores on the STAIC-T were regressed hierarchically onto either RCMAS-P or FSSC-R scores in step 1, or then, CASI scores in step 2. Scores on the CASI predicted additional variance in trait anxiety for younger, $\Delta R^{2}=.16$ and .12 , and older children, $\Delta \mathrm{R}^{2}=.09$ and .16 , for the models using the RCMAS-P and FSSC-R respectively. To test the hypothesis that CASI would predict additional variance in fear scores beyond trait anxiety in both age groups, FSSC-R scores were regressed hierarchically onto STAIC-T scores, anxiety symptom frequency, and CASI scores. The CASI was found to predict additional variance in fear beyond trait anxiety and anxiety, $\Delta R^{2}=.10$ and .12 , for the younger and older samples respectively.

To test whether age moderated the prediction of trait anxiety from CASI scores, Weems et al. (1998) also conducted separate multiple regression analyses predicting STAIC-T scores, from FSCC-R scores, CASI scores, age and either the product term of age and CASI (to test for linear moderation) or age-squared and CASI (to test for curvilinear moderation). Neither interaction term was found to be significant. Finally, the authors presented the partial correlations between CASI and FSSC-R scores, as well as the predicted covariate-adjusted slope of the CASI on FSSC-R scores by age, controlling for STAIC-T scores in both instances. Results did not indicate a trend with respect to age. Altogether, these results present confirmation that the CASI exhibits incremental validity over trait anxiety in youth.

Muris et al. (2001) examined the incremental validity of the CASI with the same sample used to examine the factor structure of the CASI. Partial correlations were calculated between CASI scores and measures of anxiety disorder symptoms using the 
Spence Children's Anxiety Scale (SCAS; Spence, 1998), controlling for youth sex and trait anxiety (STAIC-T). Significant partial correlations were found between the CASI total score and the SCAS total score, $r=.51$; and between the CASI total score and subscales related to Generalized Anxiety Disorder (GAD), $r=.37$; Separation Anxiety Disorder (SAD), $r=.25$; Social Phobia (SOP), $r=.24$; Panic Disorder (PD) and Agoraphobia, $r=.45$; Obsessive-Compulsive Disorder (OCD), $r=.30$; and Physical Injury fears, $r=.20$.

AS, Anxiety and Depression in Youth. Taking the lead from the adult literature, the first studies involving AS and anxiety in youth examined relations between AS and anxiety disorders. Rabian, Peterson, Richters, and Jensen (1993) compared CASI scores in youth diagnosed with anxiety disorders $(n=18)$, externalizing disorders $(n=31)$, and children with no psychiatric diagnosis $(n=62)$, using analysis of variance (ANOVA) procedures. Scores on the CASI were found to be highest for youth with anxiety disorders $(M=30.56)$, next for youth with externalizing disorders $(M=28.84)$, and lowest for youth with no diagnosis $(N=26.40)$. Results indicate that AS is highest in youth with anxiety disorders.

In the first study examining the relation between AS and panic in youth, Lau, Calamari and Waraczynski (1996) found a significant correlation, $r=.42$, between AS, as measured by the CASI, and panic symptoms, as measured by the Panic Attack Questionnaire (PAQ; Norton, Dorward, \& Cox, 1986) using a community sample of high school students, ages 14 to 18 years $(M=16.74$ years). The sample was then split into "panickers" ( $n=30)$ and "non-panickers" $(n=47)$, and CASI scores were found to be significantly higher for the panickers, $M=32.20, \mathrm{SD}=4.77$, than for the non-panickers, 
$M=27.66, S D=4.36, t(76)=3.51, p<.005$. The results support findings from the adult literature, indicating a relation between AS and panic symptoms and suggesting AS to be a cognitive risk factor for panic.

In a study designed to further the assessment of the relations between AS and panic in youth, Calamari et al. (2001) evaluated the incremental validity of the CASI in predicting children's panic symptoms, as measured by the SCAS Panic-Agoraphobia subscale, using hierarchical multiple regression techniques with data from a community sample of youth $(N=52 ; M=9.48$ years, $S D=2.60)$. Analyses included gender, depression scores, as measured by the short form of the Children's Depression Inventory (CDI: Kovacs, 1992), trait anxiety using the STAIC-T), and AS using the CASI. Multiple hierarchical models were tested with AS being entered in either in step 3 or 4 (with either STAIC-T or CDI scores being entered in step 4, when CASI scores were entered in step 3). In each model, CASI scores were found to predict additional variance in panic symptoms, $\Delta R^{2}=.12$ to .40 , providing further evidence for AS as a risk factor for panic disorder in youth.

Research has also suggested a relation between AS and depression (Weems et al., 1997; Joiner et. al, 2002; Lambert, McCreary et al., 2004). Following research conducted on AS and depression with adults (Otto et al. 1995), Weems et al. conducted the first study systematically examining the relations between AS and depression in a clinic sample of youth ( $N=234$; ages 6 to $17, M=10$ years, 4 months). Relations between anxiety, depression, and AS were examined using correlations and partial correlations between RCMAS total and subscale scores, Worry-Oversensitivity, Physiological, and Concentration; CDI total scores; and CASI total scores. Significant correlations were 
found between the Worry-Oversensitivity, Physiological, and Concentration subscales of the RCMAS and the CASI, $r=.63, .48$ and .55 , respectively; and between the CDI score and the CASI, $r=.52$. A series of partial correlations were also conducted using CASI total scores, CDI total scores, RCMAS subscale and total scores, and clinician ratings of severity of primary diagnosis, as measured by the Anxiety Disorders Interview Schedule for Children (ADIS-C/P: Silverman \& Nelles, 1988). The correlations between the RCMAS total score and the CASI remained significant after controlling for severity, CDI scores, and all combinations of the RCMAS subscales scores, except when the combination of the Physiological and Worry-Oversensitivity subscales were controlled for. The correlation between the CDI total score and the CASI remained significant after controlling for severity, RCMAS total scores, and all combinations of the RCMAS subscales. This study represented an important first step in linking AS to depression in the youth literature.

Now that research had begun to elucidate the link between AS, anxiety and depression in youth, the next logical step was to evaluate the facets of AS as they related to anxiety and depression. In the first study to examine these relations, Joiner et al. (2002) used a measure of youth AS devised by Laurent, Schmidt, Catanzaro, Joiner, and Kelly (1998) from the ASI, with an inpatient sample of 47 youth ages 9 to 17 years $(M=14.23$ years, $S D=1.89$ ). In this measure of AS, there are only two lower-order factors, Fear of Physical Arousal (10 items; e.g., It scares me when my heart beats fast) and Phrenophobia (3 items; e.g., I worry that I am going crazy when I can't keep my mind on something). Separate hierarchical multiple regressions were used to examine the relations of AS to anxiety symptoms, as measured by the RCMAS, controlling for either trait 
anxiety alone, as measured by the STAIC-T; or depressive symptoms alone, as measured by the CDI; or both trait anxiety and depressive symptoms. In all models, AS predicted RCMAS scores controlling for STAIC-T and CDI scores. In addition, AS accounted for between 9 and $12 \%$ of additional variance in the prediction of anxiety scores, beyond trait anxiety and depressive symptoms.

Next, separate hierarchical multiple regressions were used to examine the relations of AS to depression, while controlling for anxiety, using the same procedures described above. After controlling for anxiety, AS scores did not significantly predict CDI scores. Using a similar set of regression equations, the relations between the facets of AS - Phrenophobia and Fear of Physical Arousal - and anxiety and depression were explored. Both the Fear of Physical Arousal and Phrenophobia scales were found to predict RCMAS scores, after controlling for depression scores; $t(44)=2.98, p<.05 ; t$ (44) $=2.74, p<.01$, respectively. Neither the Fear of Physical Arousal nor the Phrenophobia scales were found to predict CDI scores, after controlling for anxiety scores; $t(44)=-0.28, p>.05 ; t(44)=0.04, p>.05$, respectively.

These results conflicted with findings in the literature (Otto et al., 1995; Weems et al. 1997) that found a significant relation between AS and depression using correlational analyses. It is important to note that sample studied by Joiner et al. (2002) may not have been large enough to find a significant relation between AS and depression if the corresponding effect size was small to moderate. In addition, the authors note that the reliability of the Phrenophobia scale was low, $\alpha=.56$. Finally, because the authors used the AS measure devised by Laurent et al. (1998), the three- or four- factor CASI more commonly used in the literature were not able to be examined. 
In the study involving a community sample of urban, African-American adolescents $(N=679 ; 12$ to 15 years, $M=13.8$ years, $S D=0.37)$, Lambert, McCreary et al. (2004) sought to further clarify the relations of the specificity of the facets of AS to both anxiety and depression. The ASI was used to measure anxiety, and to be consistent with the existing literature with regard to AS and African-Americans, the authors chose to use 4 factor structure of the ASI found by Carter et al. (1995) with African American college students, Fear of Cardiovascular Sensations, Fear of Unsteadiness, Phrenophobia and Emotional Control. The factor structure proposed by Carter et al. was verified to fit the adolescent sample through CFAs, however the internal consistency of the Emotional Control subscale was poor, $\alpha=.28$. As such, analyses were conducted only with the first three subscales listed above. The Baltimore How I Feel scale (BHIF; Ialongo, Kellam \& Poduska, 1999), a measure designed for an epidemiological study of DSM-III-R disorders in youth, was used to measure anxiety and depression symptoms. Items on the BHIF were drawn from the CDI, RCMAS, and SCAS rating scales, among others. Hierarchical multiple regressions were used to examine the relations between the anxiety and depression BHIF subscales and both the ASI subscale and total scores.

During a priori comparisons, girls were found to score significantly higher on the BHIF anxiety and depression subscales than boys. As such, youth sex was included in all hierarchical regression models. Results of regressions indicated that the AS total score predicted BHIF anxiety scores after controlling for youth sex in step 1, and BHIF depression scores in step 2. Results also indicated that the AS total score predicted BHIF depression scores after controlling for youth sex in step 1, and BHIF anxiety scores in step 2. However, the relationship was negative indicating that when AS decreased, 
depression scores increased, when controlling for youth sex and BHIF anxiety scores. The authors note that ASI total scores account for $20 \%$ of the remaining variance in BHIF anxiety scores, but only $1 \%$ of the remaining variance in BHIF depression scores.

To examine specificity of the facets of AS and anxiety and depression, Lambert, McCreary et al. (2004) conducted separate hierarchical regression with either BHIF anxiety or depression scores as the dependent variable. In each case, youth sex was entered into the model at step 1, either the BHIF depression or anxiety score was entered in step 2, Phrenophobia was entered in step 3, and both the Fear of Cardiovascular Sensations and the Fear of Unsteadiness scores were entered in step 4, to represent the fear of physical sensations as a whole. The variables entered in step 4 accounted for additional variance in BHIF anxiety scores $\Delta R^{2}=.027, p<.001$ ), when controlling for youth sex and BHIF depression scores in steps 1 and 3, and Phrenophobia scores at step 3 $\left(\Delta R^{2}=.065, p<.001\right)$. With respect to depression, the variables entered in step 4 accounted for additional variance in BHIF depressiøn $\left(R^{2}=.010, p<.01\right)$, when controlling for youth sex and BHIF anxiety scores in steps 1 and 2 and Phrenophobia scores at step $3\left(\Delta R^{2}=0.0, p>.05\right)$. The Fear of Cardiovascular sensations $(\beta=-.111, p<$ .01 ) accounted for the change in $R^{2}$, as the Fear of Unsteadiness was not significantly related to BHIF depression scores $(\beta=-.003, p>.05)$. While the addition of the Phrenophobia in step 3 did not account for additional variance in depression scores, Phrenophobia was significantly associated with BHIF depression scores $(\beta=.08, p<.05)$ in the final model.

Overall, results indicated that AS, as a unitary construct, showed specificity to anxiety and that all of the facets of AS were significantly positively related to anxiety. 
The relation between AS and depression appears to be more complex. Phrenophobia was significantly positively related to depression, Fear of Cardiovascular sensations was significantly negatively related to depression, while Fear of Unsteadiness was not significantly related to depression. These results contradict the finding of Joiner et al. (2002) as they indicate that specific components of AS are differentially related to both anxiety and depression in youth.

Only one study has examined the relations of the four facets of AS as measured by the CASI with anxiety and depression. Using a clinic sample of adolescents with internalizing disorders $(N=185 ; M=15.09$ years, $S D=1.09)$, Dia \& Bradshaw (2008) evaluated the relations between the facets of AS, anxiety and depression, using the CASI and the Revised Child Anxiety and Depression Scale (RCADS; Chorpita, Yim, Moffit, Umemoto \& Frances, 2000). The RCADS subscales are designed to correspond with the internalizing disorders, and includes subscale scores for GAD, PD, SAD, SOP, OCD and Major Depression (MDD).

Six separate regressions were conducted with the anxiety and depression subscale used as dependent variables. Hierarchical models were evaluated with age and youth sex entered in step 1, a measure of negative affectivity entered in step 2 (PANAS-C; Laurent et al., 1999), and the specific facets of AS being entered in step 3. The authors included negative affectivity in the model because empirical evidence indicates it is a common predictor of anxiety and depression. Also, for each individual model, the authors chose to enter only those facets of AS that were hypothesized to correspond with each disorder based on findings in the adult literature. 
For GAD, only the Mental Incapacitation Concerns and Social Concerns facets of AS were examined. Results indicated that only Mental Incapacitation Concerns significantly predicted GAD scores after controlling for age, youth sex and negative affectivity $(B=1.2, p<.05)$, with higher levels of Mental Incapacitation Concerns predicting higher GAD scores. For SOP, only the Social Concerns facet of AS was examined. Results indicated that Social Concerns significantly predicted SOP scores after controlling for age, youth sex and NA $(B=1.79, p<.01)$, with higher levels of Social Concerns predicting higher GAD scores. For SAD, only the Disease Concerns and Unsteady Concerns facets of AS were examined. Results indicated that only Disease Concerns significantly predicted SAD scores after controlling for age, youth sex and NA ( $B=1.05, p<.01)$, with higher levels of Disease Concerns predicting higher SAD scores. For PD, only the Disease Concerns and Unsteady Concerns facets of AS were examined. Results indicated that both the Disease Concerns $(B=1.5, p<.01)$ and Unsteady Concerns $(B=1.15, p<.01)$ facets significantly predicted SAD scores after controlling for age, youth sex and negative affectivity with higher levels of Disease Concerns and Unsteady Concerns predicting higher PD scores. For MDD, only the Mental Incapacitation Concerns facet of AS was included in the model, along with positive and negative affectivity as measured by the PANAS-C. ${ }^{2}$ Results indicated that Mental Incapacitation Concerns was not significantly associated with depression scores after controlling for age, youth sex and both positive and negative affectivity.

${ }^{2}$ In the study by Dia \& Bradshaw (2008), positive affectivity was not included in models involving anxiety as the dependent variable. The author's decision to include a measure of positive affectivity only in models involving depression as the outcome was guided by past research. 
Taken together, these results extend the findings of Laurent, McCreary et al. (2004), as they indicate the specificity of the AS factors with respect to the different anxiety disorders. The results relating AS to depression are similar to the results found by Joiner et al. (2004), as they do not provide support for a relation between the facets of AS and depression.

Summary. The literature has clearly documented relations between AS and anxiety in youth. In fact, evidence is beginning to accumulate linking specific facets of AS with the different anxiety disorders. The relations between AS and depression in youth remains less obvious. Two studies have found support for a link, while two other studies have reported no link. Further research is needed to help clarify these relations.

\section{Meta analyses with $A S$}

As the interest on the role of AS has increased within the psychological community and evidence linking AS to anxiety and depression has continued to accumulate with both adult and youth samples, two meta-analyses have been published within the past year that have empirically evaluated differences in AS with regard to anxiety disorders and mood disorders (Olatjuni \& Wolitsky-Taylor, 2009; NaragonGainey, 2010). These studies have made important contributions to literature as they provide a comprehensive, systematic analysis of the available literature (mostly with adult samples) clarifying the relations of both the total scores and facets of AS within the internalizing disorders. As a consistent pattern of findings regarding the relations of AS to anxiety and depression has emerged from these meta-analyses, their results are presented here as a quantitative description of the most recent literature. Because research on the relations between AS and anxiety and depression is scarce in the youth literature. 
The results from these meta-analyses served as a guide in the formulation of hypothesis for the current study.

In the first meta-analysis, Olatjuni and Wolitsky-Taylor (2009) evaluated AS in three groups: participants with anxiety disorders (AD), mood disorder controls (MDC) and non-clinical controls (NCC). Studies published in peer reviewed, English language journals were included if researchers reported data from one of three versions of the ASI or the CASI for one of the groups listed above, and included at least one group meeting criteria for an anxiety or mood disorder. The meta-analysis included 38 studies ( 3 of which used the CASI to measure AS) published between 1998 and 2008, with 20,146 participants, $(M=32.91$ years; $S D=11.03)$. The authors used Cohen's $d$ as the index of effect size, with small, medium and large effect sizes being represented by $d$ values of $0.2,0.5$, and 0.8 respectively. Both a priori and exploratory comparisons were made; and, moderation analyses were conducted involving age, youth sex and version of the AS measure.

For the first of the a priori comparisons, using 22 studies to compare AS levels in the AD and NCC groups, Olatjuni and Wolitsky-Taylor (2009) reported a large effect, with the AD group reporting significantly higher AS than the NCC group, $d=$ $1.61, p<.001$. This finding was moderated by youth sex and age, with an increase in female participants and an increase in mean age being associated with larger group differences Within these 22 studies, 5 studies reported data for each of the subscales, but only the Social Concerns and Mental Incapacitation Concerns subscales could be analyzed, because of differences in measures of AS. Large effects were found for both subscales, $d=1.30$ and 1.73, $p<.001$, for Social Concerns and Mental Incapacitation 
Concerns respectively; with the AD group scoring higher than the NCC group. Next, using 11 studies to compare AS levels in the AD and MDC groups, Olatjuni and Wolitsky-Taylor reported a moderate effect, with the $\mathrm{AD}$ group reporting significantly higher AS than the MDC group, $d=0.54, p<.05$. Age, youth sex and version of the AS measure were found to moderate these effects. An increase in female participants was associated with a smaller difference between the groups; an increase in mean age of the sample increased the difference between the groups; and larger effects were observed for studies using the ASI-R than those using the ASI and CASI. Using 5 studies to compare both control groups, the authors reported a moderate effect, with the MDC group reporting significantly higher AS than the NCC group, $d=0.71, p<.001$. Too few studies presented subscale data to allow for subscale comparisons between the AD and MDC groups and the MDC and NCC groups.

For exploratory comparisons, the authors compared AS levels in participants with specific anxiety disorders (PD, SOP, PTSD, and GAD) to the NCC group. For each comparison, large effects were reported, $d=1.33$ to $2.58 ; p<.001$, with the participants with anxiety disorders reporting significantly greater AS than the NCC group. Olatjuni and Wolitsky-Taylor (2009) also compared the subscales of AS in subjects with specific anxiety disorders with that of the NCC group if the number of studies was sufficient to allow for comparisons. Again only, the Social Concerns and Mental Incapacitation Concerns subscales could be evaluated. Large effects were found when comparing both the Social Concerns and Mental Incapacitation Concerns subscales of participants with panic disorder (PD), $d=1.71,2.25, p<.001$ PTSD $, d=1.42,2.35, p<.001$; and GAD, $d=0.85, p<.001 ; d=1.03, p<.05$, as compared to the NCC group. 
In addition, Olatjuni and Wolitsky-Taylor (2009) compared AS levels in participants s with specific anxiety disorders to the MDC group. A large effect was found only when comparing participants with $\mathrm{PD}$ to the MDC group, $d=.85, p<.01$. In addition, moderating effects were found for each comparison listed above, with similar patterns regarding females and older participants emerging. Too few studies presented subscale data to allow for subscale comparisons between the PD and MDC groups.

Olatjuni and Wolitsky-Taylor (2009) also compared levels of AS, using both total scores and subscale scores, within anxiety disorder diagnoses. Only comparisons of interest to the present study are reported. Moderate effects were found when comparing participants with PD to participants $\mathrm{s}$ with GAD, OCD, SOP and specific phobia (SP; $d=$ $0.70,0.61,0.49,0.62$ respectively), with PD participants reporting higher levels of AS. No significant overall AS differences were found when comparing, the SOP and GAD groups $(d=-0.04, p=.70)$, the SOP and OCD groups $(d=0.03, p=.70)$, the SOP and SP groups $(d=0.05, p=.66)$, and the GAD and SP groups $(d=-0.19, p=.23)$. Comparisons between the anxiety disorders were made using AS subscales when possible. The SOP group reported higher Social Concerns scores than both the PD $(d=$ $0.55, p<0.01)$ and GAD groups $(d=0.65, \mathrm{p}<.05)$.

The results reported by Olatjuni and Wolitzky-Taylor (2009) present a consistent pattern of findings with respect to AS, anxiety and depression. Participants with anxiety disorders reported higher levels of AS than both the MDC group and the NCC group, with the MDC group reporting higher levels of AS than the NCC group. Most anxiety disorder groups did not differ from the MDC group with respect to AS, except for the PD group. In addition, age and youth sex were found to consistently 
moderate group differences with respect to AS, with female and older subjects typically reporting higher levels of AS. Finally, the authors state that a multidimensional evaluation of AS may improve understanding about the relation between AS and internalizing psychopathology.

In the most recent meta-analysis, Naragon-Gainey (2010) examined the specificity and magnitude of the relations of AS to both diagnoses and symptoms of the anxiety disorders and depression. Searches were also limited to peer reviewed, English language journals, and samples from adult populations, ages 18 and older. For comparisons using diagnostic-level data, studies were included if researchers reported data from the original, English language version of the ASI only; and if anxiety or depressive disorders were diagnosed via clinical interview. For comparisons using correlational symptom-level data, studies were included if researchers reported data from one of the various versions of the ASI; and, if the questionnaires used to establish anxiety or depressive symptoms had demonstrated adequate convergent and divergent validity in the literature.

First, Naragon-Gainey (2010) examined mean ASI score by diagnostic group (i.e., PTSD, GAD, PD, MDD, SOP, OCD, and SP). All mean ASI scores were significantly higher than normative community sample AS scores. An overall Q test for differences among groups was significant, $\chi^{2}(6)=564, p<.001$, and post-hoc comparisons were made using Bonferonni adjustments. These comparisons indicated that the PTSD and GAD groups had significantly higher AS scores than all other groups, though the GAD group score was not significantly different from the PD group score. The MDD and SOP groups reported lower AS scores than PTSD, PD and GAD groups, 
but higher AS scores than the SP group. The OCD group score did not significantly differ from the SOP or the SP group.

Next, the author examined correlations (corrected for unreliability with coefficient alphas from both measures being correlated) between the AS total scores and symptoms of anxiety and depression by comparing the magnitude of mean correlations across symptom types. These results parallel those found in the previous analyses, indicating robustness in the reported relations. Panic and GAD symptoms were most strongly correlated with AS ( $\rho s=.60$ and .58 respectively). The correlation of AS with PTSD symptoms $(\rho=.54)$ was weaker than the correlation between AS and panic, but not significantly different from that of GAD. Symptoms of MDD, agoraphobia, SOP and OCD ( $\rho s=.45$ to .49$)$ were moderately associated with AS, having weaker associations that the previously listed symptoms. SP symptoms had the weakest association with AS $(\rho s=.40)$.

Naragon-Gainey (2010) then reported relations between three lower-order factors of the AS - Mental Incapacitation Concerns, Social Concerns and Physical Concerns - and anxiety and depression symptoms. Facets of AS were compared within each symptom type and comparisons relevant to the present study are reported. The Mental Incapacitation Concerns $(\rho=.50)$ and Physical Concerns $(\rho=.54)$ scales of AS were more strongly associated with PD than the Social Concerns $(\rho=.40)$ scale. For GAD symptoms, the correlations with the Social Concerns $(\rho=.54)$ and Mental Incapacitation Concerns $(\rho=.56)$ facets were stronger than correlations with the Physical Concerns facet $(\rho=.47)$. The Social Concerns $(\rho=.50)$ facet of AS was more strongly associated with SOP than the Physical Concerns $(\rho=.27)$ and Mental Incapacitation 
Concerns $(\rho=.38)$ facets.

Naragon-Gainey (2010) also report that for SP, the facets of AS were more loosely correlated with symptoms. The Physical Concerns $(\rho=.36)$ and SP symptom correlation was stronger than the Mental Incapacitation Concerns $(\rho=.29)$ and Social Concerns correlations $(\rho=.30)$. For MDD symptoms, the Mental Incapacitation Concerns $(\rho=.53)$ component was more strongly correlated to AS than both the Physical Concerns $(\rho=.40)$ and Social Concerns scales $(\rho=.38)$.

Subsequently, select disorders were broken down into "symptom dimension groups" and correlations with the higher and lower-order facets of AS were examined. Only relations between AS and SOP and AS and MDD will be discussed. For SOP, the symptom groups of general and performance social anxiety were examined. Results indicated that performance social anxiety $(\rho=.61)$ was more strongly correlated with higher-order AS than general social anxiety symptoms $(\rho=.50)$. For the performance symptom subgroup, the correlation with the Social Concerns facet was the strongest ( $\rho=$ .62), while correlations with Physical Concerns and Mental Incapacitation Concerns subscales were significant, but weaker ( $\rho=.45$ and 51, respectively). For the general symptom subgroup, the correlation with the Social Concerns facet was the strongest ( $\rho=$ $.55)$, followed by the correlation with Mental Incapacitation Concerns $(\rho=.43)$, which was also significantly stronger than the correlation with Physical Concerns $(\rho=.28)$.

For MDD, analyses were conducted with the following symptom groups: dysphoria, suicidality, lassitude, insomnia, appetite loss, appetite gain and well-being. The symptom dimensions of suicidality, lassitude and insomnia were moderately correlated with higher-order AS $(\rho=.39-.54)$, while the dysphoria dimension was 
highly correlated with higher-order AS $(\rho=.61)$. The correlations between higher-order AS and the remaining symptom dimension were less than .30. With respect to the lowerorder factors, the dysphoria component was most strongly correlated with the Mental Incapacitation Concerns facet $(\rho=.63)$, though the relations with the Social Concerns and Physical Concerns facets $(\rho=.51$ and .51$)$ were still strong. The suicidality component also was most strongly correlated with the Mental Incapacitation Concerns facet $(\rho=.52)$, though the relations with the Social Concerns and Physical Concerns facets ( $\rho=.36$ and .40 respectively) were still significant. Within the lassitude symptom dimension, correlations with the AS facets ranged from .35 to .42 , while correlations with the insomnia dimension and AS facets ranged from .34 to .41 . The correlations with the remaining symptom dimensions and AS facets were all less than .33.

Finally, the relations of higher-order AS and internalizing symptoms were evaluated within the context of a structural model. Using structural equation modeling (SEM) techniques, a two-factor latent variable model of internalizing symptoms was created, with one factor consisting of "fear disorders" (i.e., PD, Agoraphobia, SOP and SP) and the other factor consisting of "distress disorders" (i.e., GAD, PTSD, depression). ${ }^{3}$ Naragon-Gainey (2010) tested two models: one in which paths from AS loaded onto general factors and another in which paths from AS loaded onto individual disorders. The models were estimated using the correlation matrix for the symptoms related to the disorders and the correlations of AS with each of the symptom types.

The first model showed borderline fit to the data $(\mathrm{CFI}=.939$, SRMR $=.043)$, while the second model fit the data well $(\mathrm{CFI}=.974, \mathrm{SRMR}=.033)$. A nested chi-square

\footnotetext{
${ }^{3}$ Naragon-Gainey (2010) also tested a single-factor latent variable model in which fear disorder and distress disorders were combined into one factor. This model did not fit the data well.
} 
comparing the models indicated that the second model fit the data best, $\chi^{2}(5)=941.73, p$ $<.001$. The fear and distress higher-order factors were highly correlated $(r=.73)$, while the individual disorders fit the factors well, with factor loadings ranging from .71 to .86 . The paths from AS to the various disorders were compared using confidence intervals, and comparisons were adjusted using a Bonferonni correction $(p<.0012)$. The higherorder AS factor was most strongly related to agoraphobia, PD, GAD and PTSD $(r=.79$ to .92). Moderate relations were indentified between AS and SOP $(r=.74)$ and between AS and MDD $(r=.67)$, though the correlation between AS and PTSD did not significantly differ from the correlation between AS and SOP. The relation between AS and SP was the weakest $(r=.63)$. The findings from the path analyses also mirror the findings from the diagnostic group and correlational analyses reported earlier.

In sum, the results presented by Naragon-Gainey (2010) provide the most detailed portrayal of the relations of AS, anxiety and depression to date. Results indicate that PD and GAD have the strongest relations to AS, while SOP and MDD are moderately related to AS. The weakest relation between AS and anxiety was consistently found between SP and AS. With respect to the facets of AS, both Physical Concerns and Mental Incapacitation Concerns were strongly associated with PD, indicating that is not only the fear of physical symptoms that is related to PD, but also the fear of cognitive dyscontrol. All 3 facets of AS were strongly related to GAD, which is in line with the general scope of the disorder. SOP seems to be specifically related to the Social Concerns facet of AS, while MDD is most strongly related to the cognitive dyscontrol facet of AS. This is the first study to examine the relations of symptoms of MDD with the facets of AS, and results indicate that the Mental Incapacitation Concerns facet of AS is most 
strongly related to the dysphoria aspect of depression. This is also the first study to examine the AS internalizing relations using SEM techniques. Results indicated that even after controlling for shared variance among the disorders, AS was still significantly associated with all of the fear and distress disorders, including depression

Summary

The study of AS in youth has come a long way since the concept was first proposed by Reiss (1985). The distinction between AS and trait anxiety in youth has been demonstrated empirically (Chorpita, Albano, \& Barlow, 1996). Psychometric research on AS has demonstrated that AS is not a unitary construct as once thought, but rather a multifaceted construct (Silverman et al., 2003). Additional research has provided an external validation of these facets by indicating that individual AS factors differentially related to various types of anxiety and possibly to depression (Joiner et al., 2002; Lambert, McCreary et al., 2004).

Several important questions remain, however. First, the factor structure of the CASI, the most commonly used measure to assess AS in youth, should be evaluated further. Although the four-factor model proposed by Silverman et al. (2003) has the strongest basis for support in the literature, its utility with minority groups remains in question. The only study in literature to examine the factor structure of the CASI with minorities involved African-American youth (Lambert, Cooley, et al., 2004), and the 4factor model provided a poor fit to the data. To date, a study has not been published examining the factor structure of the CASI with Hispanic-Latino youth. As HispanicLatinos represent the largest and fastest growing minority group in the US (approximately 48.5 million; United States Census Bureau, 2009), research on anxiety 
and its risk factors utilizing Hispanic samples is beginning to accumulate (Pina \& Silverman, 2004; Varela, Weems, Berman, Hensley \& Rodriguez de Bernal, 2007; Varela \& Hensley-Maloney, 2009). As cultural bias can methodically influence the measurement of a construct (Knight \& Hill, 1998), it is necessary to determine that equivalent factor structures of the CASI exist across ethnic groups before conducting research using the CASI with various ethnic populations in order to avoid making false statements.

Second, the findings of three studies mainly focused on assessing the relations between the facets of AS and anxiety and depression in youth provide very different pictures of these relations. These differences might be associated with sample size and characteristics (e.g., clinic vs. non-clinic; ethnicity), methodology used to evaluate the relations (e.g., correlation analyses vs. regression analyses), or differences in the rating scales used to assess the AS, anxiety and depression. The ability to identify cognitive risk-factors, such as AS, that are specific to different types of internalizing psychopathology would be beneficial from both a research and a clinical perspective. Clarifying these relations would advance etiological models of the internalizing disorders. In addition, this clarification would allow for a more focused development of both prevention and intervention strategies aimed at cognitive risk factors (Dia \& Bradshaw, 2008 ; Hayward et al., 2000,), especially in light of the success of recent prevention studies specifically targeting reductions in AS (Schmidt et al., 2007).

Thus, conducting additional research examining the specific factor structure of AS across groups and examining the relation of specifics facets of AS to anxiety and depression in youth would not only help researchers refine developmental models of psychopathology but would also help clinicians to develop more focused treatment 
interventions. This represents the aim of the present dissertation study.

Present Study

The main objective of the present study is to clarify the relations between AS's facets with anxiety and depression, in a large sample of Hispanic-Latino and EuropeanAmerican children and adolescents, herein referred to as youth, referred to a universitybased anxiety specialty clinic. Various structural models involving AS (as measured by the CASI), anxiety (as measured by the RCMAS), and depression (as measured by the CDI) will be examined. These models estimate anxiety and depression scores simultaneously while accounting for the shared residual variance between the constructs.

Based on the literature reviewed above, I hypothesized the CASI total score would significantly predict RCMAS scores, after shared residual variance with CDI scores was accounted for. In addition, I hypothesized CASI total scores would significantly predict CDI scores after shared residual variance with RCMAS scores was accounted for. In addition, I hypothesized that all facets of AS would significantly predict RCMAS total scores, while controlling for shared residual variance in CDI scores. However based on the results from the meta-analyses described above, only the Mental Incapacitation Concerns facet of AS was hypothesized to significantly predict CDI total scores, while controlling for shared residual variance in RCMAS scores.

In light of the findings by Naragon-Gainey (2010) that facets of AS show specificity to symptom dimensions of the internalizing disorders, the relations of the facets of AS to the various symptom dimensions of anxiety and depression (as measured by the RCMAS and CDI) and were explored. Most of the subscales of RCMAS and CDI did not directly map onto the symptom dimension anxiety scores measured by Naragon- 
Gainey. In these cases, the analyses are considered exploratory and no hypotheses were formulated. However, one of the CDI subscales, Dysphoria, directly related to the Dysphoria depression symptom dimension scale analyzed by Naragon-Gainey. In this case, I hypothesized that the results will be consistent with those of the meta-analysis and the Mental Incapacitation Concerns facet of AS would be significantly related to the Dysphoria subscale of the CDI.

Finally, because the exact factor structure of the AS construct is still in question, before examining the structural models listed above, I formally examined the factor structure of AS using the 18-item version of the Childhood Anxiety Sensitivity Index (CASI: Silverman, Fleisig, Rabian, \& Petersen, 1991) to determine which of the proposed structures discussed in the extant literature best fits the current sample. Specifically, I tested the three- and four-factor models presented by Silverman et al. (2003). Given that the sample used in the present study is largely comprised of Hispanic-Latino youth, the cross-ethnic equivalence of the CASI between Hispanic-Latino and European-American youth was examined using ordered-categorical multiple-group CFA procedures. Because the clinic sample used in the present study is similar to a portion of the combined sample used by Silverman et al. in their CFA of the CASI, I hypothesized that the four-factor structure found by Silverman et al. would provide the best fit to the data. Because of the lack of past empirical research regarding the factor structure of the CASI with HispanicLatino youth, I made no specific hypothesis regarding the cross-ethnic equivalence of the CASI. 


\section{CHAPTER III}

\section{METHODOLOGY}

\section{Participants}

Participants were 730 youth (47.3\% girls), 7 to 16 years of age $(\mathrm{M}=10.15$ years, $\mathrm{SD}=2.34)$, referred to an anxiety disorders specialty research clinic. In terms of ethnicity, 164 (22.5\%) were European-American and 566 (77.5\%) were Hispanic-Latino. One-hundred and four families (14.2\%) reported annual incomes of \$20,999 or less; 137 (18.8\%) reported incomes between $\$ 21,000$ and $\$ 40,999 ; 306(41.9 \%)$ reported incomes over \$41,000; and 183 families (25\%) did not report income (see Table 2). Fifty-four percent of Latino youth were born in the US; $13.4 \%$ were born in Caribbean, Central American, or South American countries (e.g., Cuba, Puerto Rico, Nicaragua, Mexico, Venezuela, Peru, and Colombia). The remaining 32.5\% did not report their country of birth.

Youth were included if their parent reported them to have difficulties related to anxiety symptoms during an initial telephone screen. Exclusionary criteria included severe psychopathology (e.g., schizophrenia) or developmental delays (e.g., Autism, Asperger's syndrome, mental retardation). Of the 664 youth (90.9\%) for which diagnoses were obtained, 613 met DSM-IV diagnostic criteria for the presence of a primary anxiety disorder in their diagnostic profile. In addition, 85 youth met DSM-IV criteria for a depressive disorder in their diagnostic profile. Approximately five percent of youth did not complete the semi-structured diagnostic interview used to determine diagnoses (ADIS C/P); however, because these youth completed the questionnaires, their data were included. The most common primary diagnoses were Separation Anxiety 
Disorder (32.1\%), Social Phobia (15.2\%), Specific Phobia (14.4\%), and Generalized Anxiety Disorder (13.2\%). Of the 730 youth, 562 (77\%) had at least one comorbid disorder. Sociodemographic information by ethnicity is presented in Table 2 .

Measures

Anxiety Disorders Interview Schedule for DSM-IV: Child and Parent Versions (ADIS-IV: C/P; Silverman and Albano, 1996). The ADIS-IV: C/P is a semi-structured diagnostic interview schedule designed specifically for the diagnosis of anxiety disorders in children and adolescents and other related disorders. Test-retest reliability of the ADIS-C/P has been reported to be in the good to excellent range (Silverman, Saavedra and Pina, 2001). To determine diagnoses, clinicians conducted separate interviews using the child and parent versions, respectively, of the ADIS-IV: C/P. The information obtained from the child and the parent interviews were combined to reach a combined diagnosis. In the case of a discrepancy between the child and parent interviews, if one or both interviews yielded a diagnosis with an interference rating of four or more (on a $0-8$ point rating scale), the child received the diagnosis and was assigned the higher of the two interference ratings. In cases of multiple diagnoses, the relative impact or interference of each specific diagnosis was used as the basis for assigning the primary diagnosis, the secondary diagnosis, etc. This includes diagnoses for all disorders - not just anxiety, which can be reliably differentiated using the ADIS-C/P interviews (Silverman and Albano, 1996).

Childhood Anxiety Sensitivity Index (CASI; Silverman et al., 1991). The CASI was used to assess AS and consists of 18 items that assess the extent that youth view the experience of anxiety related symptoms as aversive (e.g., "It scares me when I feel 
shaky"). The CASI is a modified version of the Anxiety Sensitivity Index (ASI; Peterson \& Reiss, 1987) with an additional two items. Respondents rate their agreement to each item along an ordinal scale of 1 (None) to 3 (A lot). Total scores range from 18 to 54, with higher scores indicating higher levels of AS. Silverman et al. (1991) reported testretest reliability estimates (using a 2 week retest interval) of .79 and .76 for clinical and non clinical samples, respectively, and internal consistency estimates (alpha coefficients) of .87 for both samples. Silverman et al. (2003) reported that both 3 and 4 factor models of the CASI fit the data well, with the 4 factor model providing the best fit for the data. The three-factor model includes (1) Physical Concerns (e.g., When my stomach hurts, I worry that I might be really sick), (2) Mental Incapacitation Concerns (e.g., When I am afraid, I worry that I might be crazy and (3) Social Concerns (e.g., I don't want others to know I'm afraid). The four-factor model breaks down Physical Concerns into (3) Disease Concerns (e.g., It scares me when my heart beats fast), and (4) Unsteady Concerns (e.g., It scares me when I feel faint). Both factor structures will be evaluated in the present study; and, the factor structure providing the best fit to the data will be used for all subsequent analyses.

Revised Children's Manifest Anxiety Scale (RCMAS; Reynolds \& Richmond, 1978). The RCMAS consists of 37 total items. Twenty-eight of the items assess youths' anxiety symptoms and the remaining 9 items make up a Lie scale. Respondents rate each item with either Yes or No. Total Anxiety Scale scores range from 0 to 28. Pela and Reynolds (1982) reported the RCMAS to have a test-retest reliability estimate of .98 . Reynolds and Richmond (1985) reported an internal consistency (alpha) coefficient of .80 for the Total Anxiety Scale score. Pina, Little, Knight, and Silverman (2009) reported 
metric invariance of the RCMAS across age, gender and ethnicity (Hispanic-Latino vs. European-American) using a sample similar to the present one. The scale consists of three symptom factors, Physiological symptoms, Worry/Oversensitivity, and Concentration.

Children's Depression Inventory (CDI; Kovacs, 1992). The CDI contains 27 items that consists of three statements of different severity ratings and requires the child to choose one statement that best describes him or her. Each item is scored from 0 to 2 and the sum of all item scores yields the total CDI score. Scores range from 0 to 54 . Internal consistencies of the CDI have been reported as ranging from .71 to .91 (Carle, Millsap \& Cole, 2008). Concurrent validity has been demonstrated by finding significant correlations between the CDI and clinicians' independent global depression ratings (e.g., $\mathrm{r}=.55$; Kovacs, 1992). The factor structure identified by Craighead, Smucker, Craighead and Ilardi (1998), and subsequently confirmed by Carle, Millsap and Cole (2008), consists of 5 symptom factors, Dysphoria, Externalizing, Self-Depreciation, School Problems and Social Problems. Carle et al. reported metric invariance across youth sex for the Craighead 5 factor model with a community sample of youth ages 7 to 13 . In addition, Pina et al. (2008) found metric invariance of the CDI across age, youth sex and ethnicity (Hispanic-Latinos vs. European-Americans) using a sample similar to the present one. ${ }^{4}$

\footnotetext{
${ }^{4}$ Prior to evaluating the fit of models involving the CDI, measurement equivalence of the five-factor CDI model proposed by Craighead et al. (2008) was examine across age, sex and ethnicity. Analyses supported metric equivalence of CDI across age, sex and ethnicity.
} 


\section{Procedure}

All youth and parents first provided informed consent/assent to participate in the assessment phase of one of three clinical trials taking place at a university based child anxiety clinic. Subsequently, the youth were administered a semi-structured diagnostic interview (ADIS C/P) and a battery of questionnaires, including the CASI, RCMAS, and CDI. The questionnaires were administered by trained graduate or advanced undergraduate research assistants. Prior to completion of each questionnaire, directions for each were read aloud. Individual questionnaire items were read aloud to younger children, as well as to youth with reading difficulties, with the youth reading along with the research assistant (who was instructed not to view the youth's responses to reduce the possibility of demand). After the youths completed the questionnaire battery, the trained research assistant checked the questionnaire battery for response sets and answer omissions.

\section{Diagnosticians}

The majority of the interviews were conducted by graduate students in psychology, with a few being conducted by the first author of the ADIS C/P (Silverman and Albano, 1996). All diagnosticians were extensively trained in handling circumstances that may arise during the interview of child and their parents, with careful attention being paid to assessing the interference ratings and ranking of the diagnoses. Training involved participation in numerous clinical meetings concerning the administration of the interview schedule. Pertinent issues involving the diagnosis of DSM-IV anxiety, depression, externalizing and other related childhood disorders were also discussed. Additionally, the diagnostician was required to observe five separate parent and child 
interviews and "match" on five consecutive diagnoses. Moreover, the diagnostician was required to conduct and interview under observation and "match" the diagnoses. In order to "match" correctly, the diagnostician had to agree on all specific diagnoses given based on the parent interview, child interview and composite diagnoses. Lastly, agreement was contingent upon order of diagnoses as well (primary, secondary, tertiary etc.).

\section{Assessment and Diagnosis}

After obtaining informed consent from both the child and parent, the ADIS-C and ADIS-P were administered with the order of administration being randomly determined. While the interview was being administered to one informant, the other participant was completing the required questionnaires. Participants were allowed to schedule another session if the interview or questionnaires were not completed during the first session. The diagnostician did not discuss, with either participant, information or details provided by the other informant, in order to avoid biasing the informant's responses.

Upon completion of the initial assessment, the diagnostician derived DSM-IV diagnoses from the child interview, the parent interview and combined interview data according to the procedures defined in the ADIS C/P clinician manual (Silverman \& Albano, 1996). The initial diagnoses were then presented at a weekly clinical meeting to other certified ADIS-C/P diagnosticians and the first author of the interview in order to come to a final diagnosis based on a consensus model. Diagnoses were listed in order of interference (primary, secondary) and were given corresponding interference ratings on a nine-point scale ( 0 to 8 ). In addition, the diagnostician provided information pertaining to exactly how the DSM-IV diagnosed disorder interfered with the child's everyday functioning in the major areas of life (school, friends and family life, internal distress) as 
recounted by both the child and parent. After a brief discussion, during which the participants of the meeting were allowed to ask specific questions pertaining to the nature of symptoms or interference, diagnoses were finalized an approved by the first author of the ADIS-C/P. Children who met criteria for a primary anxiety disorder as determined by the final diagnosis approved in the clinical meeting were randomly assigned a therapist.

\section{Data Analysis}

Frequencies and Descriptive Statistics. Descriptive statistics were computed on demographic variables and variables relevant to the models to provide a sense of the characteristics of the sample using SPSS (version 17). In addition, Cronbach's alphas for each of the subscales used in models were computed as a measure of internal consistency.

Factor Structure of the CASI. CFAs using ordered categorical variable methodology (CFA-OCM, Muthén \& Asparouhov, 2002; Millsap \& Yun-Tein, 2004) were preformed on the CASI using Mplus (version 6.0) to evaluate both the three- and four-factor models of the 18-item CASI as proposed by Silverman et al. (2003) as shown in Figure 1. Since the items on the CASI are ordinal in nature, when conducting itemlevel factor analysis of such items, categorical variable methodology was applied in order to avoid problems resulting from the application of traditional continuous factor methodology such as underestimated parameter and overestimated standard errors. Only the 713 participants who completed at least $70 \%$ of the items on the CASI were included in the analyses. ${ }^{5}$ The fit of the models was evaluated using the Weighted Least Squares

\footnotetext{
${ }^{5}$ The participants excluded from analyses $(n=17)$ were missing all 18 items of the CASI. Six hundred and ninety-eight of the remaining 713 participants completed all 18 -items of the CASI. The remaining $2 \%$ of participants completed the majority of the 18-items. No participant had more than 3 items missing. Each item had less than $1 \%$ of scores missing. As missing data were very minimal, an EM algorithm using Amelia II was used to impute missing data for those 15 participants with missing items.
} 
algorithm (WLSMV) with delta parameterization (Muthén \& Asparouhov, 2002; Millsap $\&$ Yun-Tein, 2004) which is reasonably robust to violations of non-normality.

In WLSMV, coefficients are estimated in a similar way to probit regression and WLSMV is appropriate for use with categorical data (Muthén \& Muthén, 2007). In CFAs using categorical variable methodology (Millsap \& Yun-Tein, 2004; Cole et al., 2008), several measurement parameters are estimated or constrained, including intercepts, factor loadings, thresholds and uniqueness. The intercepts represent the value of the items when the value of its specified latent factor is zero, while the loadings represent how much each item is related to its specified latent factor. Uniqueness reflects the variance in each item not attributed to its specified latent factor, or error. Each of these parameters is also estimated when using traditional ML continuous CFA techniques. The threshold parameters are unique to CFA-OCM models and mirror the ordered categorical properties of the individual items. The thresholds represent specific values on an underlying latent response variant. For any given individual, if the value of the variant is below the threshold for a given item, the individual would respond in one category; if the value of the variant is above the threshold for a given item, then the individual would respond in the next category, and so on (Cole et al., 2008). The standardized thresholds map onto quantiles of the standard normal distribution (Muthén \& Asparouhov, 2002).

To evaluate the fit of the proposed models of the CASI on the current sample, a variety of global fit indices were used, including indices of absolute fit, indices of relative fit and indices of fit with a penalty function for lack of parsimony, following the recommendations of Bollen and Long (1993). The overall chi-square test of model fit was used, with non- significant values indicating good model fit. However, non-significant 
chi-square values are not likely to be obtained with large sample sizes (Hu \& Bentler, 1999). Thus, a variety of other fit indices were given precedence to the chi square estimate. These fit indices include the CFI, which should be greater than .95; TLI, which should be greater than .95; and the RMSEA, which should be less than .08 for satisfactory fit (Hu \& Bentler, 1999). In addition to the global fit indices, more focused tests of fit were pursued. These included examination of modification indices, which should be less than 4.00, and examination of parameter estimates for Heywood cases. Nested model testing with WLSMV algorithms (see Muthén and Muthén, 2007) were used to compare the fit of the three- and four-factor models of the CASI. ${ }^{6}$

Metric Equivalence of the CASI. Multiple group CFA-OCMs using the WLSMV estimator with theta parameterization (Millsap \& Yun-Tein, 2004) in Mplus (Version 6.0) were conducted to evaluate the configural and metric invariance of the factor model found to provide the best fit for the current sample across Hispanic-Latinos and European-Americans. Millsap and Yun-Tein state that the theta parameterization is favored over the delta parameterization in multiple group solutions as theta allows researchers to test the hypothesis that the error variances differ across groups, while the delta method does not.

To test for configural invariance, a CFA-OCM model was tested in which thresholds and factor loadings were permitted to be free across groups. For the reference

\footnotetext{
${ }^{6}$ The difference in chi-square values for two nested models using the WLSMV chi-square values is not distributed as chi-square. The WLSMV chi-square difference test compares the null analysis model to a less restrictive alternative model in which the null model is nested. To obtain a correct chi-square difference test when using WLSMV, a two step procedure is performed within Mplus. In the first step, the alternative model is estimated and the derivatives needed for the chi-square difference test are saved. In the second step, the null model is estimated and the chi-square difference test is computed using the derivatives from both analyses (Muthén \& Muthén, 2007).
} 
group, factor means were fixed at zero, variances were estimated, and the residuals were fixed at 1.0. For the remaining group, factor means, variances and residuals were estimated. For statistical identification, the factor loadings for the first item of each factor (a marker variable) was fixed to one across groups; the first threshold of each observed variable was held invariant across groups; for the marker variable, an additional threshold value was required to be invariant across the groups; and one additional threshold value was required to be invariant across the groups for each additional factor in the model. Next, to test for metric invariance, a model was evaluated in which factor loadings were constrained to be equal across groups, while retaining constraints specified in the configural invariance model. Then, retaining the constraints in the previous model, a model was tested in which thresholds were constrained to be equal across groups. Finally, a model was tested in which uniqueness was constrained to be equal across groups, while retaining the constraints in the previous model. Nested model testing with WLSMV algorithms (see Muthén \& Muthén, 2007) was used to compare the CFA model with more stringent constraints to the model with lesser constraints at each step of the process.

$A S$, Anxiety, and Depression. To evaluate the relations between AS, anxiety and depression, three structural models were examined with SEM techniques using Mplus (version 6.0). Each model was evaluated using the MLR estimator which produces maximum likelihood parameter estimates with robust standard errors based on the HuberWhite sandwich estimator and a chi-square test statistic based on a comparable algorithm (Muthén \& Muthén, 2006).

In the first model, the RCMAS and CDI total scores were regressed onto CASI total scores. In the second model, the RCMAS and CDI total scores were regressed onto 
each of the factors of the CASI. In the third model, the RCMAS and CDI subscale scores were regressed onto each of the factors of the CASI.

For each model, ethnicity and youth sex (reflected by dummy variables), as well as age, were included as covariates in the prediction of RCMAS and CDI scores. In addition, error terms associated with the RCMAS and CDI total and subscale scores were allowed to be correlated with each other. As the models evaluated were just-identified, fit indices could not be examined. For each model, parameter estimates were examined to determine the relations between AS, anxiety and depression.

Missing Data, Outliers, and Non-normality. As missing data were minimal for most variables (e.g., less than $4.1 \%$ of the cases on a single variable and less than $5.1 \%$ of the cases have at least one missing value), those values that were missing were imputed using Expectation-Maximization (EM) based procedures as provided by Amelia II (version 1.2-17; Honaker, King \& Blackwell, 2010). This is full information imputation strategy which produces less bias in the imputation estimates than traditional listwise deletion procedures (Acock, 2005). Missing data bias was assessed by computing a dummy variable reflecting the presence or absence of missing data for each variable in the model. This dummy variable was correlated with all other variables in the model as well as an array of demographic variables. No significant correlations were found $(\mathrm{p}>.05)$.

Non-model based outlier analyses were undertaken prior to all major analyses. Multivariate outliers were identified by examining leverage indices for each individual and defining an outlier as a leverage score four times greater than the mean leverage statistic. When evaluating structural models, model based outliers were examined by 
computing standardized dfbeta statistics and defining an outlier as having a standardized dfbeta greater than 1.0. No outliers were found. In addition, univariate indices of skewness and kurtosis were examined to determine if the absolute value of any of these indices is greater than 2.0. As the examination of these indices revealed non-normality within the data (see Table 3), only estimators robust to violations of non-normality (e.g., WLSMV and MLR) were used for all analyses. 


\section{CHAPTER IV}

\section{RESULTS}

\section{Descriptive Statistics}

A summary of the descriptive statistics, including means, standard deviations, skewness and kurtosis, for relevant variables are presented in Table 3. Skewness ranged from -0.21 to 1.90 , while kurtosis ranged from -1.01 to 4.42 . Nonnormality was present for two of the five CDI subscales. Cronbach's alpha coefficients for each subscale used in subsequent models are ranged from .55 to .90 .

\section{Factor structure of the CASI}

A series of confirmatory factor analyses were conducted to determine the most appropriate factor structure of the CASI among a clinic-referred sample of Hispanic and European-American anxious youth. Specifically, goodness-of-fit indices were examined for the 3 and 4 factor models of the 18-item CASI proposed by Silverman et al. (2003) presented in Figure 1. In addition, nested model testing with WLSMV algorithms was performed to evaluate the fit of the 3 and 4 factor models against one another.

Table 4 presents the fit indices of the 3 and 4 factor models of the 18-item CASI. Both the three and four-factor models provided good fit to the data. The nested chi-square difference test revealed no significant difference between the four-factor model and three-factor models, $\chi^{2} \Delta(3)=7.60, p=.06$. When examining the parameter estimates obtained with the four-factor model, the factors of Disease Concerns and Unsteady concerns were correlated 0.95 . As such, the decision was made to use more parsimonious three-factor CASI model presented in Figure 1. However, upon examination of the parameter estimates of the three-factor model, it was revealed that the factors of Physical 
Concerns and Mental Incapacitation Concerns were correlated 0.86. As such, the decision was made to collapse these factors and examine the fit of a two-factor model made up of a Social Concerns factor and a factor combining Physical and Mental Incapacitation Concerns. While a nested chi-square difference test revealed a significant difference between the three-factor model and the two-factor models, $\chi^{2} \Delta(2)=36.84, p<.001$, with the three-factor model providing a better fit for the data, the two-factor model also fit the data well (see Table 4). The decision was ultimately made to use a two-factor CASI model despite the results from chi-square difference test, as it is impacted by the large sample size. In addition, the sizeable correlation between the two factors in the three factor model creates problems for empirical tests differentiating the two. The two factors of Social Concerns factor and Physical/Mental Concerns in the final two factor model were correlated .56 .

\section{Metric Invariance of the CASI}

After establishing that a two-factor model was the most appropriate model, the configural invariance of the CASI across European-Americans and Hispanic-Latinos was tested $^{7}$. Though the chi-square for the configural invariance model was statistically significant, $\chi^{2}(270)=490.12$; the remaining fit indices suggested excellent fit to the data, RMSEA $=0.048 ; \mathrm{CFI}=0.972 ; \mathrm{TLI}=0.968$, supporting the hypothesis of configural invariance. Next, a model was fit to the data to test for invariant loadings, in which factor loadings were constrained to equality across groups, while retaining the constraints imposed in the configural invariance model. Again, though the chi-square for the model with invariant loadings was statistically significant, $\chi^{2}(286)=508.36$, the remaining fit

\footnotetext{
${ }^{7}$ Prior to evaluating the measurement equivalence of the CASI across ethnicity, analyses supported metric equivalence of CASI across sex and age.
} 
indices suggested excellent fit to the data, $\mathrm{RMSEA}=0.047 ; \mathrm{CFI}=0.972 ; \mathrm{TLI}=0.970$. The adjusted chi-square difference test, $\chi^{2} \Delta(16)=26.45, p=.048$, was significant, however the amount of departure from invariance is minor and the statistically significant result is most likely driven by a large sample size. Cheung and Rensvold (2002) suggest comparing CFIs between two models when evaluating measurement invariance rather than examining chi-square difference tests to circumvent the issues created by large sample sizes and model complexity when using chi-square difference tests. They suggest that a CFI difference smaller than or equal to 0.01 indicates that the null hypothesis of invariance should not be rejected. A change in CFI is independent of model complexity and sample size and is not correlated with overall fit measures. As the CFIs for the configural invariance model and the invariant loading model were equal, the hypothesis of invariant loadings was supported.

Next, a model was fit to the data to test for invariant thresholds, in which thresholds were constrained to equality across groups, while retaining the constraints imposed in the factorial invariance model. Though the chi-square for the model with invariant thresholds was statistically significant, $\chi^{2}(300)=508.79$, the remaining fit indices suggested excellent fit to the data, RMSEA $=0.044 ; \mathrm{CFI}=0.973 ; \mathrm{TLI}=0.973$. The adjusted chi-square difference test, $\chi^{2} \Delta(14)=12.42, p=.57$ and the change in CFI supported the hypothesis of invariant thresholds. Finally, a model was fit to the data to test for invariant uniqueness, in which uniqueness of items were constrained to equality across groups, while retaining the constraints imposed in the threshold invariance model. Though the chi-square for the model with invariant uniqueness was statistically significant, $\chi^{2}(318)=476.96$, the remaining fit indices suggested excellent fit to the data, 
RMSEA $=0.037 ; \mathrm{CFI}=0.980 ; \mathrm{TLI}=0.980$. The adjusted chi-square difference test, $\chi^{2} \Delta$ $(18)=16.70, p=.54$, and the change in CFI supported the hypothesis of invariant uniqueness. Taken together, the results support the measurement invariance hypothesis.

As the measurement invariance hypothesis was supported, Tables 5 and 6 summarize the standardized parameter estimates for the two-factor model of the CASI for all participants estimated with WLSMV and the delta parameterization. Standardized factor loadings ranged from .52 to .80 . Threshold values describing movement from none to some ranged from -1.02 to 0.40 , whereas threshold values describing movement from some to a lot ranged from -0.01 to 1.23 . Item residual variances or item uniqueness ranged from .36 to .73 .

AS, Anxiety, and Depression

In order to evaluate the relations between AS, anxiety and depression, three different models were evaluated using the sample covariance matrix as input. Because non-normality was present with two of the CDI subscales, the MLR estimator, which is robust to violations of normality, was used. The models were just identified.

CASI Total Scores with RCMAS and CDI Total Scores. In the first model tested, the RCMAS and CDI total scores were regressed onto CASI total scores (see Figure 2). Residual terms associated with the RCMAS and CDI scores were correlated. Age, youth sex and ethnicity were included as covariates for RCMAS, CDI and CASI scores. The model was just-identified.

Figure 2 presents relevant unstandardized coefficients obtained from the first model. The residuals indicate the proportion of unexplained variance in the endogenous 
variables. The model as specified was able to account for $35 \%$ of the variance in RCMAS total scores and $26 \%$ of the variance in CDI total scores.

As Figure 2 shows, AS significantly predicted RCMAS total scores, $B=0.48, S E$ $=.02, p<.001,95 \% C I=.44$ to .51 . AS was also a significant predictor of CDI total scores, $B=0.50, \mathrm{SE}=.03, p<.001,95 \% C I=0.44$ to 0.55. In both cases, higher CASI total scores predicted higher anxiety scores and higher depression scores.

CASI Subscale scores with RCMAS and CDI Total Scores. In the second model tested, the RCMAS and CDI total scores were regressed onto the two CASI subscale scores confirmed with the current sample: Physical/Mental Concerns and Social Concerns (see Figure 3). Residual terms associated with the RCMAS and CDI scores were correlated. Age, youth sex and ethnicity were included as covariates for RCMAS and CDI total scores. This model was just identified.

Figure 3 presents relevant unstandardized coefficients obtained from the second model. The residuals indicate the proportion of unexplained variance in the endogenous variables. The model as specified was able to account for $35 \%$ of the variance in RCMAS total scores and $26 \%$ of the variance in CDI total scores. The two facets of AS significantly predicted RCMAS total scores, $B=0.47, S E=.03, p<.001,95 \% C I=0.42$ to $0.51 ; B=0.50, S E=.14, p<.001,95 \% C I=0.27$ to 0.72 ; for Physical/Mental Concerns and Social Concerns respectively. In each case, higher scores on the CASI subscales predicted higher anxiety. Only the Physical/Mental Concerns facet of AS significantly predicted CDI total scores, $B=0.53, S E=.04, p<.001,95 \% C I=0.45$ to 0.60. Higher scores on the Physical and Mental concerns subscales predicted higher depression. Social Concerns did not significantly predict depression. 
CASI Subscale scores with RCMAS and CDI Subscale Scores. In the third model tested, the three RCMAS subscale scores and the five CDI subscale scores identified by Craighead et al. (1998) were regressed onto the two CASI subscale scores (see Figure 4). Residual terms associated with all three RCMAS and five CDI subscale scores were correlated. Age, youth sex and ethnicity were included as covariates for all RCMAS and CDI subscales. This model was just identified.

Figure 4 presents relevant unstandardized coefficients obtained from the second model. For the RCMAS subscales, the model as specified was able to account for $23 \%$ of the variance in Physiological symptoms scores, $21 \%$ of the variance in Concentration scores, and $32 \%$ of the variance in Worry-Oversensitivity scores. For the CDI subscales, the model as specified was able to account for $7 \%$ of the variance in the Externalizing scores, $24 \%$ of the variance in the Dysphoria scores, $21 \%$ of the variance in SelfDepreciation scores, $19 \%$ of the variance in School Problems scores, and $8 \%$ of the variance in Social Problems scores.

Both facets of AS predicted RCMAS Concentration scores, $B=0.11, S E=.01$, $p<.001,95 \% C I=0.10$ to $0.13 ; B=0.12, S E=.05, p<.001,95 \% C I=0.04$ to 0.19 ; for Physical/Mental Concerns and Social Concerns respectively. In each case, higher scores on the CASI subscales predicted an increase in concentration related anxiety symptoms. Both facets of AS also predicted RCMAS Worry-Oversensitivity scores, $B=0.21, S E=$ $.01, p<.001,95 \% C I=0.19$ to $0.24 ; B=0.29, S E=.05, p<.001,95 \% C I=0.18$ to 0.41 ; for Physical/Mental Concerns and Social Concerns respectively. In each case, higher scores on the CASI subscales predicted an increase in anxiety symptoms involving concentration. Only the Physical/Mental Concerns facet of AS predicted RCMAS 
Physiological scores, $B=0.15, S E=.01, p<.001,95 \% C I=0.13$ to 0.17 . Higher scores on the Physical/Mental Concerns subscale predicted an increase in physiological anxiety symptoms. Social Concerns did not significantly predict and increase in physiological anxiety symptoms.

For depression, the Physical/Mental Concerns facet of AS significantly predicted all five CDI subscale scores, $B=0.04$ to 0.15 . In each case, higher scores on the Physical/Mental Concerns subscale predicted higher CDI subscale scores. Social Concerns did not significantly predict any of the CDI subscales. 


\section{CHAPTER V \\ DISCUSSION}

\section{Factor Structure of AS}

The results from this study of the factor structure of AS with a clinic sample of anxious youth are not consistent with prior research evaluating the factor structure of the CASI with primarily non-clinic samples of youth. With the current sample, CFAs provided support for a two-factor model of the 18-item CASI. This factor structure is different from the three- and four- factor models proposed by Silverman et al. (2003). While fit statistics indicated that the three- and four-factor models proposed by Silverman et al. fit the data well, high correlations between the Disease Concerns, Unsteady Concerns and Mental Incapacitation Concerns factors warranted the collapsing of the three factors into a single factor of all physical and mental incapacitation Concerns. This Physical/Mental Concerns factor is comprised of 15 of the $18 \mathrm{CASI}$ items and encompasses the fear of anxiety symptoms that are experienced internally, whether they be physical in nature (e.g., a fast beating heart or an upset stomach) or mental in nature (e.g., ruminations or a lack of concentration). The present study did find support for the Social Concerns factor of AS as reported by Silverman et al. This factor encompasses items that involve the fear of other people becoming aware of one's anxious feelings. Additional analyses conducted evaluating the relations between the facets of AS and the symptoms of anxiety and depression served as an external validation of the two-factor model, as the facets differentially predicted various internalizing symptoms dimensions. 
The most plausible explanation for the CASI factor structure differences found in the present study relates to the type of sample used. The majority of factor analytic studies conducted on the CASI that found support for either three- or four-factor models involved primarily non-clinic samples. Silverman et al. (2003) confirmed the factor structure of the CASI using only the Australian non-clinic subsamples. The correlations between the four factors for this part of the sample were all below .68, much lower than the correlations found with the present clinic sample. Subsequently, Silverman et al. fit the four-factor model with the entire American subsample and tested for factorial invariance across the clinic and non-clinic subsamples. Chi-square difference tests indicated that the hypothesis of equal factor loadings between clinic and non-clinic participants was tenable. However, they do not report the correlations between the factors for the two American subsamples. The possibility also exists that the facets of Physical Concerns and Mental Incapacitation Concerns are indeed two separate lower order AS constructs. However, within a clinic sample of anxious youth these constructs are so highly correlated, that it is not possible to differentiate between the two.

In the only other factor analysis conducted entirely with a clinic sample of anxious youth, Chorpita and Daleiden (2000) conducted EFAs and found a one factor solution fit for the 18 items. Interestingly enough, the 3 items with the weakest factor loadings and item-total correlations were items 1,5 and 17 , or the three items that comprise the Social Concerns factor in the model proposed in the current study. Chorpita and Daleiden went on to conduct CFAs on a two-factor model consisting of autonomic and non-autonomic factors constructed a priori by independent raters. 
With respect to the cross-ethnic equivalence of the CASI, this study is the first to demonstrate that the items on the CASI provided invariant measurement across Hispanic/Latinos and European Americans in a clinic sample of anxious youth. These results support both previous and continued cross-ethnic use of the CASI in clinical comparisons. The hypothesis that the items were patterned in the same way for both Hispanic-Latinos and European-Americans was supported by various fit indices. Models testing invariant factor loadings, thresholds, and item uniqueness all fit the data well, and chi-square difference tests and CFI differences between successive models generally supported the hypothesis of metric invariance. In other words, for both Hispanic-Latinos and European-Americans, the items correspond to each latent factor in a similar manner (loadings), the responses to the items that correspond with movement from one category to another within each item are similar (thresholds), and the amounts of error variance associated with each item are similar (uniqueness).

The examination of the exact structure and number of AS facets, as well as the cross-ethnic equivalence of these facets, is important from a theoretical standpoint, as this clarification provided the empirical basis to examine exactly how different facets of AS related to various types of psychopathology in youth, namely anxiety and depression. AS, Anxiety, and Depression

This study represents the first study to examine structural models involving AS, anxiety, and depression in a clinic sample of anxious youth. As predicted, AS total scores accounted for a significant amount of variance in both anxiety and depression total scores. In both instances, higher levels of AS predicted increased anxiety and depression scores. These findings contradict the findings of Lambert, McCreary et al. (2004), who 
reported a negative relation between AS and depression, but support the findings of Weems et al. (1997), who reported positive associations between AS and depression. It is possible that sample differences play a role in the difference in findings, as Lambert and colleagues employed a community sample of African-American youth, while Weems et al. examined the relations with a clinic-referred sample of anxious youth similar to the present one.

Also as predicted, both facets of AS significantly predicted RCMAS total scores, while controlling for shared residual variance in CDI scores. As the high correlation between Physical Concerns and Mental Incapacitation Concerns warranted their collapse into a single factor, it was not possible to examine the individual contribution of these facets. The hypothesis regarding the relation of Mental Incapacitation Concerns to depression was not able to be evaluated. However, the Physical/Mental Concerns facet of AS significantly predicted CDI total scores, while controlling for shared residual variance in RCMAS scores. The Social Concerns facet of AS did not significantly predict depression total scores. Results indicate that while the Social Concerns facet of AS is specific to anxiety, the Physical/Mental concerns facets of AS are related to both anxiety and depression.

Results from third model tested revealed specificity with respect to AS and the anxiety and depression symptom dimensions. Both facets of AS significantly predicted Concentration- and Worry-Oversensitivity related anxiety symptoms after controlling for shared unexplained variance with depression symptoms. These results are consistent with a large body of research consistently finding a link between high AS and high anxiety. However, Social Concerns did not significantly predict Physiological anxiety 
symptoms. This result suggests that a fear of people noticing one's anxiety did not increase one's own physiological anxiety symptoms, but did increase worries and concentration related anxiety symptoms.

Again for reasons described above, it was not possible to examine the unique contribution of Physical Concerns and Mental Incapacitation Concerns with the symptom dimensions of depression. As a result, the hypothesis that Mental Incapacitation Concerns would predict Dysphoria scores could not be examined. However, Physical/Mental Concerns predicted all five depression symptom dimensions: Externalizing, Dysphoria, Self-Depreciation, School Problems and Social Problems, after controlling for shared unexplained variance with anxiety symptoms. Social Concerns did not significantly predict any of the depression symptom dimensions. Taken together, these findings support the discriminative validity of the two AS facets found in the present study. These results are also consistent with the findings Olatjuni and Wolitzky-Taylor (2009) and Naragon-Gainey (2010), indicating that a multidimensional evaluation of AS improves understanding about the relation between AS and internalizing psychopathology.

In the tested models, Social Concerns had the least predictive value of the AS facets. Past research has suggested that Social Concerns scores were highest for people with a diagnosis of Social Phobia (Olatjuni \& Wolitzky-Taylor, 2009; Silverman et al., 2003). In addition, Naragon-Gainey (2010) reported that Social Concerns has the strongest relation with the diagnosis of Social Phobia and weaker though significant relations with other anxiety disorders. As the relation between Social Concerns and Social anxiety is likely to be strong, it is possible that Social Concerns predicts Physiological symptoms of anxiety only for youth with Social Phobia, whose primary 
symptom is worry about being embarrassed in social situations.

Clinical Implications. These findings highlight that AS's relations to youth psychiatric symptoms are complex. Focusing on the facets of AS is critical to fully understand these relations. Results also suggest that clinicians may be able to tailor treatments to target specific facets of AS. It may be useful to target all facets of AS when treating anxious youth. Within the context of cognitive-behavioral therapy (CBT), in addition to using cognitive restructuring to facilitate change in cognitions related to feared stimuli (e.g., being away from parents or having to talking front of the class), it may be useful to target fearful cognitions related to fear of the anxiety symptoms themselves. In addition, results indicate that it might be useful for clinicians to target cognitions related to physical and mental incapacitations when treating youth with depression as well. Targeting AS may even have a greater impact on youth with comorbid anxiety and depression. It would be of interest in future research to determine if CBT including a focus on the reduction of AS symptoms would increase the success rates of $\mathrm{CBT}$ in youth with comorbid anxiety and depression, who typically have poorer response to treatment.

\section{Limitations and Future Directions}

A number of limitations of this study should be mentioned. First, there are limits to the generalizability of this study's findings, as all models were examined with a clinic sample of Hispanic-Latino and European-American youth. Most previous research supported a 4-factor structure of the CASI with community samples of youth (Adornetto et al., 2008; Silverman et al., 2003). It is not known if the results found regarding the factor structure the CASI with the current sample are a result of sample characteristics. It 
is also unclear if the cross-ethnic equivalence of said structure, or the symptom specificity of AS would generalize to other samples. Previous research has indicated that the four-factor structure of the CASI did not fit with data from a community sample of African-American youth (Lambert, Cooley et al., 2004). Future research should compare the factor structure of the CASI with both clinic and non-clinic samples of various ethnicities, including European-American, Hispanic-Latinos and African-American youth and examine cross-ethnic metric equivalence with such a sample.

In addition, other types of measurement invariance, namely, functional and scalar, were not examined. According to Knight and Zerr (2010), a measure is said to have functional equivalence if similar antecedents, consequents, and association exist across groups. Scalar equivalence, which has been identified as the most important type of equivalence, is present when a score on a measure refers to the same magnitude of the construct across groups. Therefore, both functional and scalar equivalence represent integral pieces of the measurement invariance puzzle (Vandenberg\& Lance, 2000). Both should be explored in future research.

Further, although previous research has found that AS facets are uniquely related to anxiety and depression in community samples of varying ethnic composition (Dia \& Bradshaw, 2008; Lambert, McCreary, et al., 2004), the relations reported in these studies differ from the relations found in the present study. Future research is needed to continue to clarify the picture of the relations between AS, anxiety and depression to examine if these relations differ in clinic and non-clinic samples. In addition, as the current sample participants were referred for primary anxiety, it is not known if the relations among AS, 
anxiety and depression would be similar within a sample of youth referred for primary depression.

This study employed a cross-sectional design. As such, it is not possible to draw conclusions about the invariance of the factor structure of AS across time. Future research should examine this notion because if latent variable factor scores measured at different time points are established to be on the same metric, stronger conclusions can be made regarding results using those factor scores (Widaman, Ferrer, \& Conger, 2010).

Moreover, it is not possible to make causal inferences regarding the relations among AS, anxiety, and depression. McLaughlin and Hatzenbuehler (2009) examined the prospective relations of AS, anxiety, depression and stressful life events in a large community sample of adolescents. Results indicated that although AS was associated longitudinally with anxiety symptoms, it was not associated longitudinally with depression symptoms, when anxiety was included in the model. McLaughlin and Hatzenbuehler state that their results indicated that cross-sectional studies finding a link between AS and depression may actually be obscure the association between AS and depression during rapid developmental periods such as adolescence. Future studies should continue to evaluate the relations among AS, anxiety and depression prospectively to advance developmental models of internalizing psychopathology.

In sum, the current study identified the best fitting factor structure of the CASI with a clinic sample and determined the cross-ethnic equivalence of the CASI across Hispanic-Latino and European-American youth. Specific facets of AS were found to differentially predict anxiety and depression symptom dimensions. All facets of AS predicted Concentration and Worry-Oversensitivity symptoms related to anxiety. 
Physiological anxiety symptoms were predicted by Physical/Mental Concerns but not by Social Concerns. Physical/Mental Concerns was found to predict all five symptom dimensions of depression. 
Table 1

Factor Analytic Studies of the Childhood Anxiety Sensitivity Index

\begin{tabular}{|c|c|c|c|c|c|}
\hline Author(s) & Sample Charact & ristics & & $\begin{array}{l}\text { Method of } \\
\text { Factor } \\
\text { Extraction }\end{array}$ & $\begin{array}{l}\text { Number of } \\
\text { Factors }^{\mathrm{a}}\end{array}$ \\
\hline $\begin{array}{l}\text { Adornetto et al. } \\
(2008)\end{array}$ & $\begin{array}{l}4 \text { non-clinic } \\
(\mathrm{N}=1226, \\
225,230,143)\end{array}$ & $\begin{array}{l}8-16 \\
\text { years }\end{array}$ & German & EFA,CFA & 4 factors \\
\hline $\begin{array}{l}\text { Chorpita \& } \\
\text { Daleiden } \\
(2000)\end{array}$ & $\begin{array}{l}\text { clinic } \\
(\mathrm{N}=228)\end{array}$ & $\begin{array}{l}7-17 \\
\text { years }\end{array}$ & EA & EFA & 2 factors \\
\hline $\begin{array}{l}\text { Esau et al. } \\
(2010)\end{array}$ & $\begin{array}{l}\text { non-clinic } \\
(\mathrm{N}=1292)\end{array}$ & $\begin{array}{l}12-17 \\
\text { years }\end{array}$ & German & $\begin{array}{l}\text { EFA, } \\
\text { CFA }\end{array}$ & 3 factors \\
\hline $\begin{array}{l}\text { Lambert, } \\
\text { Cooley et al. } \\
(2004)\end{array}$ & $\begin{array}{l}\text { non-clinic } \\
(\mathrm{N}=144)\end{array}$ & $\begin{array}{l}8-12 \\
\text { years, }\end{array}$ & $\mathrm{AA}$ & CFA & 2 factors \\
\hline $\begin{array}{l}\text { Muris et al. } \\
(2001)\end{array}$ & $\begin{array}{l}\text { non-clinic } \\
(\mathrm{N}=819)\end{array}$ & $\begin{array}{l}13-16 \\
\text { years }\end{array}$ & Dutch & CFA & $\begin{array}{l}3 \text { or } 4 \\
\text { factors }\end{array}$ \\
\hline $\begin{array}{l}\text { Silverman et al. } \\
\text { (1999) }\end{array}$ & $\begin{array}{l}1 \text { clinic } \\
(\mathrm{N}=258) \\
1 \text { non-clinic } \\
(\mathrm{N}=249)\end{array}$ & $\begin{array}{l}7-12 \\
\text { years }\end{array}$ & EA & $\begin{array}{l}\text { EFA, } \\
\text { CFA }\end{array}$ & $\begin{array}{l}3 \text { or } 4 \\
\text { factors }\end{array}$ \\
\hline $\begin{array}{l}\text { Silverman et al. } \\
(2003)\end{array}$ & $\begin{array}{l}2 \text { non-clinic } \\
(\mathrm{N}=767,249) \\
1 \text { clinic } \\
(\mathrm{N}=258)\end{array}$ & $\begin{array}{l}7-16 \\
\text { years }\end{array}$ & $\begin{array}{l}\text { Australian, } \\
\text { EA }\end{array}$ & CFA & 4 factors \\
\hline $\begin{array}{l}\text { Walsh et al. } \\
(2004)\end{array}$ & $\begin{array}{l}\text { non-clinic, } \\
(\mathrm{N}=1698)\end{array}$ & $\begin{array}{l}7-16 \\
\text { years }\end{array}$ & Canadian & EFA & 3 factors \\
\hline
\end{tabular}

Note. $\mathrm{AA}=$ African-American, EA = European-American, EFA = exploratory factor analysis, $\mathrm{CFA}=$ confirmatory factor analysis, a. Number of factors reported refers to the number of factors that received most support by the authors. 
Table 2

Socio-demographic Characteristics of Participants

\begin{tabular}{lcl}
\hline & $\begin{array}{l}\text { European- } \\
\text { American } \\
(n=164)\end{array}$ & $\begin{array}{l}\text { Hispanic- } \\
\text { Latino } \\
(n=566)\end{array}$ \\
\hline Age, $M(S D)$ & $10.44(2.52)$ & $10.07(2.28)$ \\
Sex $($ female) $(\%)$ & 53.0 & 45.6 \\
Income $(\%)$ & & \\
$\quad<\$ 21,000$ & 9.8 & 15.5 \\
$\quad \$ 21,001$ to $\$ 40,000$ & 10.4 & 21.2 \\
$>\$ 40,000$ & 50.0 & 39.6 \\
$\quad$ Did not report & 29.9 & 23.5 \\
Most Common Primary Diagnoses $(\%)$ & & \\
$\quad$ Specific Phobia & 14.6 & 14.3 \\
$\quad$ Separation Anxiety & 36.6 & 30.7 \\
$\quad$ Social Phobia & 11.0 & 16.4 \\
$\quad$ Generalized Anxiety Disorder & 14.0 & 12.9 \\
\hline
\end{tabular}

Table 3

Means, Standard Deviations, and Relevant Estimates for all Measures

\begin{tabular}{lrccc}
\hline Scale & $M(S D)$ & Skew & Kurtosis & $\alpha$ \\
\hline CASI & & & & \\
Total & $32.12(8.01)$ & 0.39 & -0.44 & .89 \\
Physical/Mental Concerns & $25.81(7.32)$ & 0.49 & -0.46 & .90 \\
$\quad$ Social Concerns & $6.32(1.60)$ & -0.17 & -0.44 & .51 \\
RCMAS & & & & \\
Total & $12.82(6.44)$ & -0.06 & -0.77 & .88 \\
Physiological & $4.31(2.38)$ & 0.10 & -0.69 & .66 \\
Worry-Oversensitivity & $5.82(3.17)$ & -0.21 & -1.01 & .66 \\
Concentration & $2.69(1.98)$ & 0.36 & -0.90 & .70 \\
CDI & & & & \\
Total & $10.60(7.88)$ & 0.98 & 0.82 & .87 \\
Externalizing & $0.76(1.19)$ & 1.90 & 4.42 & .55 \\
Dysphoria & $2.13(2.21)$ & 1.18 & 1.00 & .71 \\
Self-Depreciation & $2.66(2.44)$ & 1.28 & 2.03 & .67 \\
School Problems & $3.39(2.59)$ & 0.61 & -0.22 & .64 \\
Social Problems & $1.66(1.60)$ & 0.86 & 0.27 & .62 \\
\hline Note. CASI = Childhood Anxiety Sensitivity Index; RCMAS $=$ Revised Children's \\
Manifest Anxiety Scale; CDI = Children's Depression Inventory; $\alpha=$ Cronbach's \\
alpha coefficient.
\end{tabular}


Table 4

Goodness-of-fit Indices for 18-item Version of CASI

\begin{tabular}{lccccc}
\multicolumn{1}{c}{ Model } & $\chi^{2}$ & $d f$ & RMSEA & CFI & TLI \\
\hline 1. Four-factor & $316.57^{* * *}$ & 129 & .045 & .977 & .972 \\
2. Three-factor & $322.47^{* * *}$ & 132 & .045 & .976 & .972 \\
3. Two-factor & $371.97^{* * *}$ & 134 & .050 & .970 & .966 \\
$\quad$ Difference between & & & & & \\
$\begin{array}{c}\text { Three- and Four- factor Models } \\
\text { Difference between }\end{array}$ & 7.60 & 3 & & & \\
Two- and Three- factor Models & $36.84^{* * *}$ & 2 & & & \\
\hline
\end{tabular}

Note. RMSEA $=$ Root Mean Square Error of Approximation; CFI $=$ Comparative Fit Index; TLI $=$ Tucker Lewis Index. ${ }^{* * *} p<.001$.

Table 5

Standardized Factor Loadings for the Two-Factor CASI Model

\begin{tabular}{|c|c|c|c|}
\hline Item & $\begin{array}{c}\text { Physical/ } \\
\text { Mental } \\
\text { Concerns }\end{array}$ & $\begin{array}{c}\text { Social } \\
\text { Concerns }\end{array}$ & $\begin{array}{c}\text { Item } \\
\text { Uniqueness }\end{array}$ \\
\hline 2. When can't schoolwork worry crazy & .65 & & .58 \\
\hline 3. Scares me when feel shaky & .72 & & .48 \\
\hline 4. Scares me when feel faint & .67 & & .56 \\
\hline 6. Scares me when heart beats fast & .73 & & .46 \\
\hline 7. Embarrasses me when stomach growls & .55 & & .70 \\
\hline 8. Scares me when feel like throwing up & 69 & & .52 \\
\hline 9. When heart fast worry something wrong & .77 & & .41 \\
\hline 10. Scares me when trouble catching breath & .64 & & .59 \\
\hline 11. When stomach hurts worry really sick & .68 & & .54 \\
\hline 12. Scares me when can't keep mind on work & .68 & & .54 \\
\hline 13. Other kids tell when I feel shaky & .53 & & .72 \\
\hline 14. Unusual feelings in body scare me & .78 & & .39 \\
\hline 15. When afraid worry might be crazy & .76 & & .42 \\
\hline 16. Scares me when feel nervous & .68 & & .54 \\
\hline 18. Funny feeling in body scares me & .80 & & .36 \\
\hline 1. Don't want others to know afraid & & .54 & .71 \\
\hline 5. Important to stay in control & & .52 & .73 \\
\hline 17. Don't like to let feelings show & & .68 & .54 \\
\hline
\end{tabular}

Note. Model estimated using WLSMV with delta parameterization. 
Table 6

Standardized Threshold Estimates for the Two-Factor CASI Model

\begin{tabular}{lrrrr}
\hline & \multicolumn{2}{c}{$\begin{array}{c}\text { Physical/ } \\
\text { Mental }\end{array}$} & \multicolumn{2}{c}{$\begin{array}{c}\text { Social } \\
\text { Concerns }\end{array}$} \\
\hline Item & \multicolumn{1}{c}{ Concerns } & 2 & 1 & 2 \\
\hline 2. When can't schoolwork worry crazy & 0.40 & 1.23 & & \\
3. Scares me when feel shaky & -0.06 & 0.89 & \\
4. Scares me when feel faint & -0.22 & 0.57 & \\
6. Scares me when heart beats fast & -0.09 & 0.77 & \\
7. Embarrasses me when stomach growls & 0.06 & 0.87 & \\
8. Scares me when feel like throwing up & -0.43 & 0.39 & & \\
9. When heart fast worry something wrong & -0.01 & 0.81 & & \\
10. Scares me when trouble catching breath & -0.32 & 0.60 & & \\
11. When stomach hurts worry really sick & -0.26 & 0.75 & & \\
12. Scares me when can't keep mind on work & 0.09 & 0.90 & & \\
13. Other kids tell when I feel shaky & 0.22 & 1.23 & & \\
14. Unusual feelings in body scare me & -0.14 & 0.87 & & \\
15. When afraid worry might be crazy & 0.43 & 1.14 & & \\
16. Scares me when feel nervous & -0.34 & 0.68 & & \\
18. Funny feeling in body scares me & 0.19 & 1.01 & & -0.58 \\
1. Don't want others to know afraid & & & & -1.02 \\
5. Important to stay in control & & & -0.54 & 0.62 \\
17. Don't like to let feelings show & & & &
\end{tabular}

Note. $1=$ Threshold estimate from none to some, $2=$ Threshold estimate from some to a lot. 


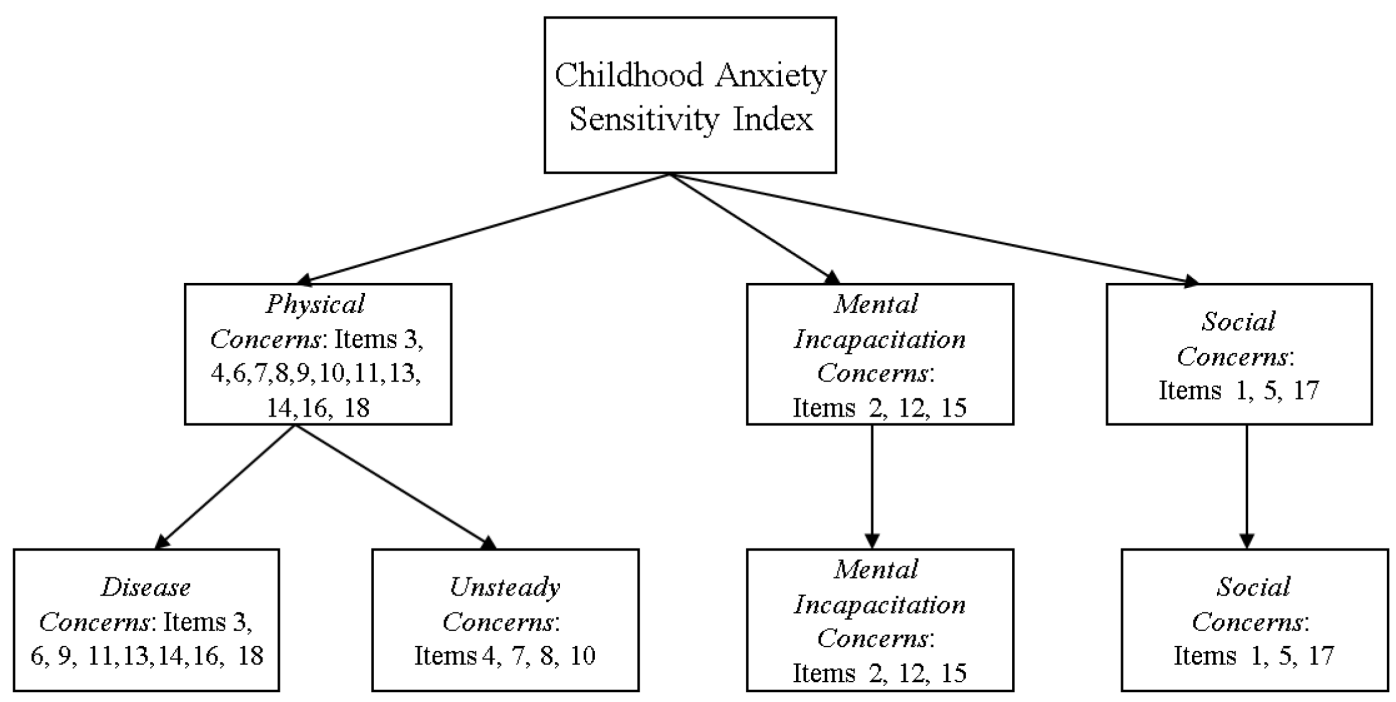

Figure 1. Classification of the 18 items of the Childhood Anxiety Sensitivity Index into three and four factors. The selection of the items loading on each factor is based largely on the results of the factor analytic study conducted by Silverman et al. (2003). 


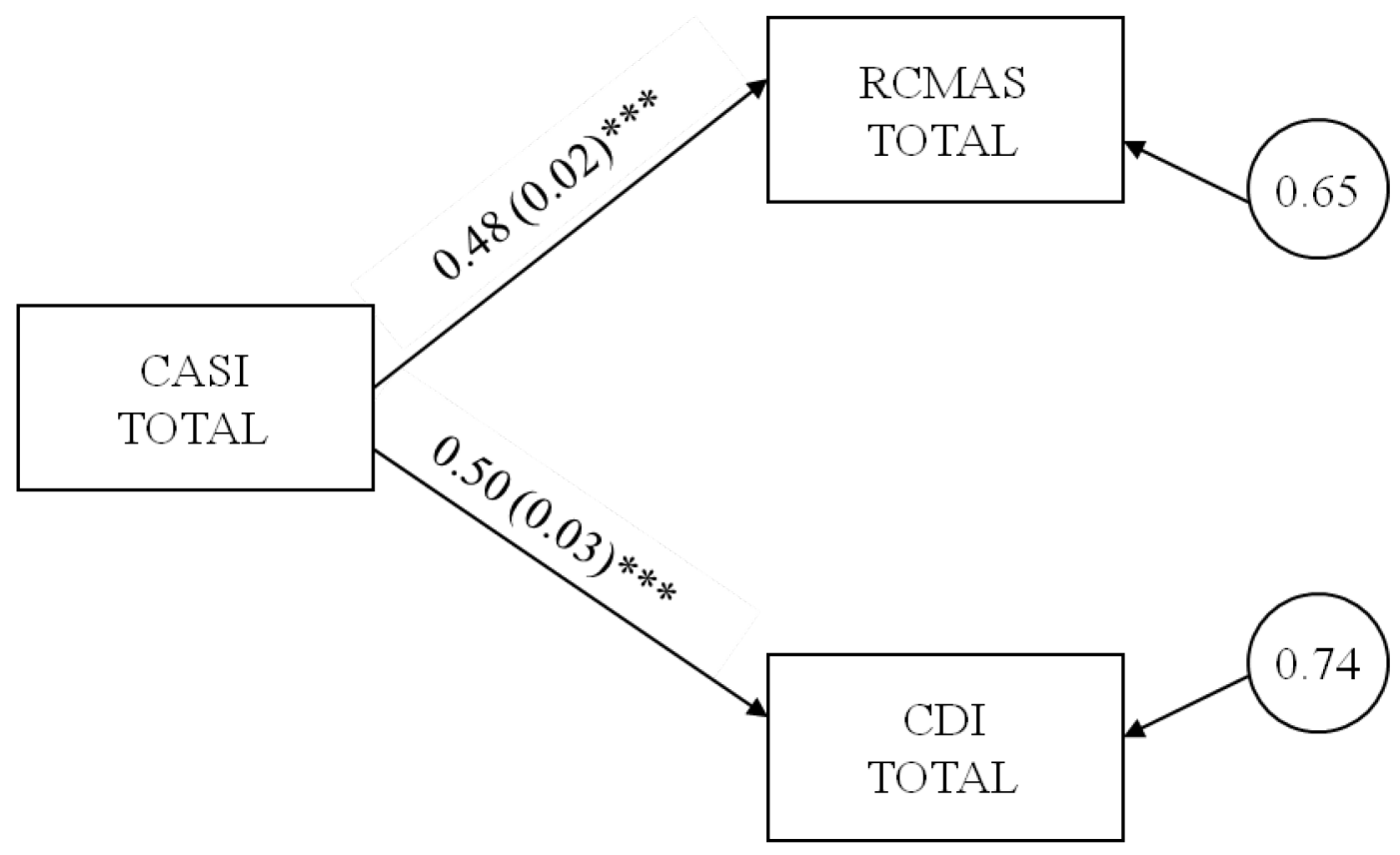

Figure 2. Relevant estimates for the model predicting anxiety and depression total scores from CASI total scores. Unstandarized path coefficients are presented with standard errors listed in parentheses. Age, youth sex and ethnicity were included as covariates for RCMAS and CDI total scores. Error terms for the RCMAS total and CDI total scores were correlated. Only significant effects for focal independent variables are included in the figure to avoid clutter. CASI = Childhood Anxiety Sensitivity Index; RCMAS = Revised Children's Manifest Anxiety Scale; CDI = Children's Depression Inventory. $* * *=\mathrm{p}<.001$ 


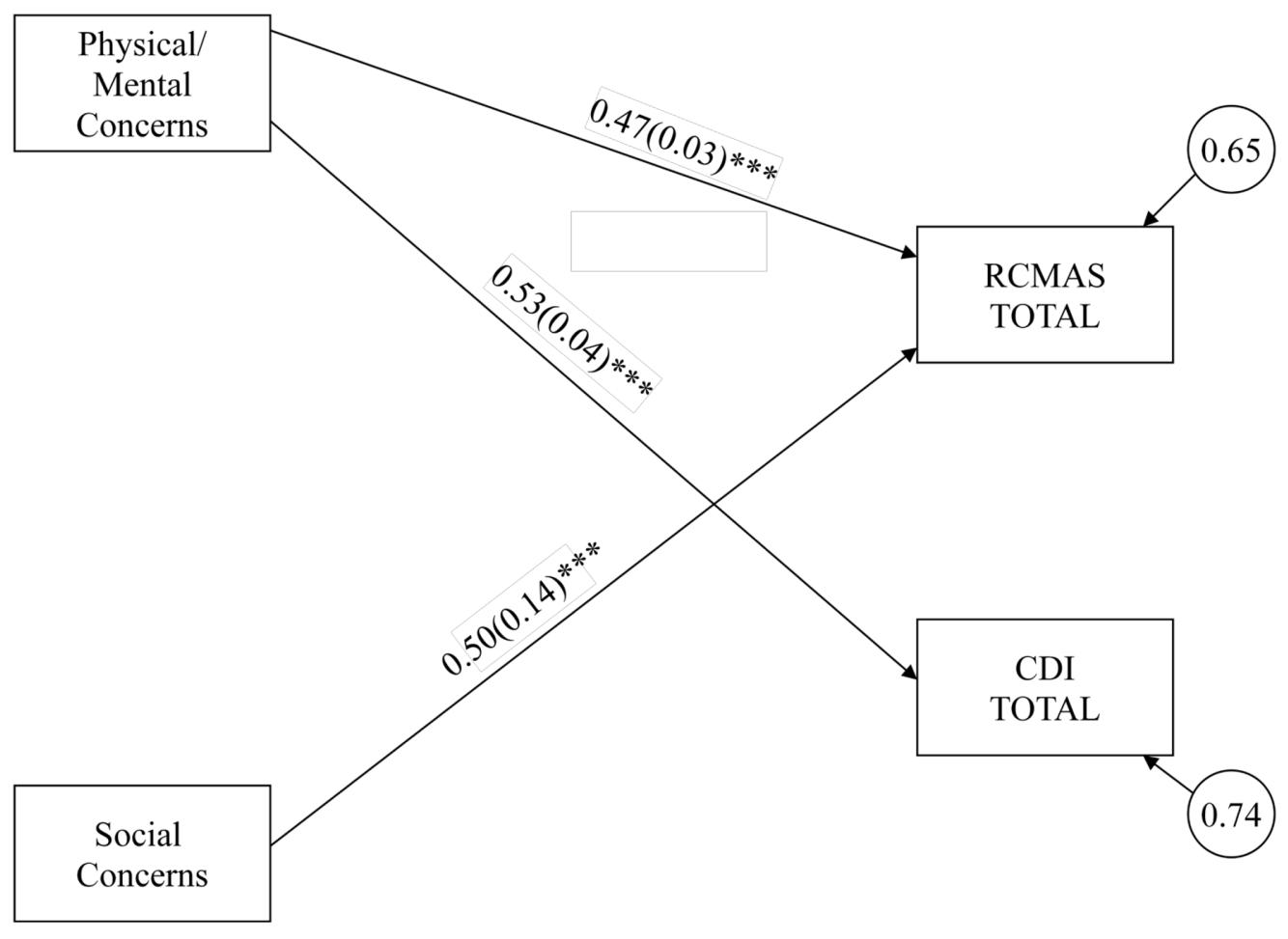

Figure 3. Relevant estimates for the model predicting anxiety and depression total scores from the four facets of AS. Unstandarized path coefficients are presented with standard errors listed in parentheses. Only significant effects for focal independent variables are included in the figure to avoid clutter. Age, youth sex and ethnicity were included as covariates for RCMAS and CDI total scores. Error terms for the RCMAS and CDI total scores were correlated. RCMAS= Revised Children's Manifest Anxiety Scale; $\mathrm{CDI}=$ Children's Depression Inventory. $* * *=\mathrm{p}<.001 ; *=\mathrm{p}<.05$ 


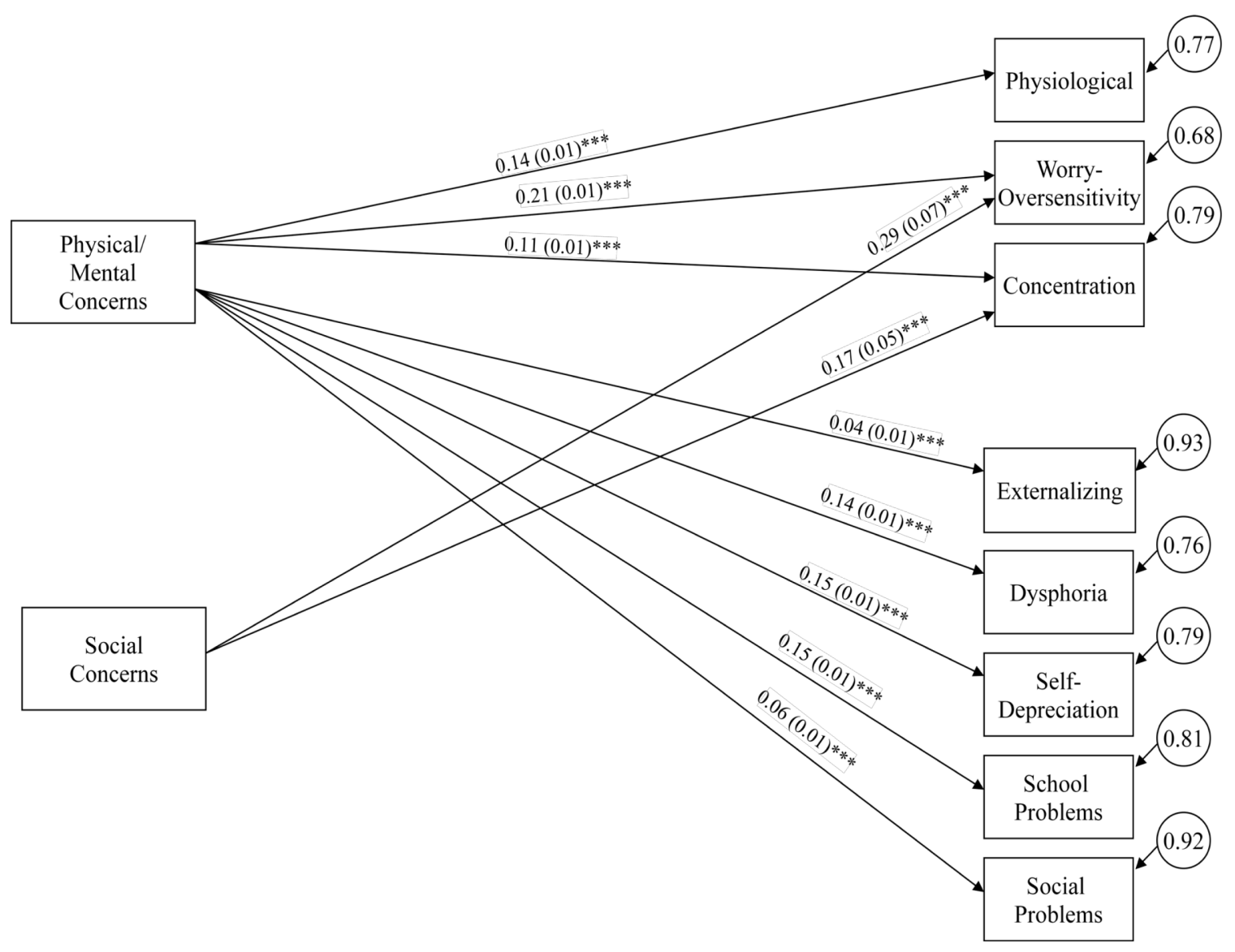

Figure 4. Relevant estimates for the model predicting anxiety and depression symptom subscale scores from the four facets of AS. Unstandarized path coefficients are presented with standard errors listed in parentheses. Only significant effects for focal independent variables are included in the figure to avoid clutter. Age, youth sex and ethnicity were included as covariates for all RCMAS and CDI subscales. Error terms for the RCMAS and CDI subscales were correlated.

$* * *=\mathrm{p}<.001$. 


\section{LIST OF REFERENCES}

Acock, A. C. (2005). Working with missing values. Journal of Marriage and Family, 67, 1012-1028.

Adornetto, C., Hensdiek, M., Meyer, A., In-Albon, T., \& Schneider, S. (2008). The factor structure of the Childhood Anxiety Sensitivity Index in German children. Journal of Behavior Therapy and Experimental Psychiatry, 39, 404-416.

Albano, A. M., \& Silverman, W. K. (1996). Guide to the Anxiety Disorders Interview Schedule for Children-IV (Child and Parent Versions). San Antonio, TX: Psychological Corporation.

Asmundson, G. J. (1999). Anxiety sensitivity and chronic pain: empirical findings, clinical implications and future directions. In S. Taylor (Ed.), Anxiety Sensitivity: Theory, Research and Treatment of the Fear of Anxiety (pp. 269-286). London: Lawrence Erlbaum Associates.

Babakus, E., Ferguson, C. E., \& Joreskog, K. G. (1987). The sensitivity of confirmatory maximum likelihood factor analysis to violations of measurement scale and distributional assumptions. Journal of Marketing Research, 37, 72-141.

Barlow, D. H. (2002). Anxiety and its Disorders: The nature and treatment of anxiety and panic (2nd ed.). New York: Guilford Press.

Bollen, K., \& Long, S. (Eds.). (1993). Testing structural equation models. Newbury Park, CA: Sage.

Calamari, J. E., Hale, L. R., Heffelfinger, A. S., Lau, J. J., Weerts, M. A., Taglione, P. A., et al. (2001). Relations between anxiety sensitivity and panic symptoms in nonreferred children and adolescents. Journal of Behavior Therapy and Experimental Psychiatry, 32, 117-136.

Carle, A. C., Millsap, R. E., \& Cole, D. A. (2008). Measurement bias across gender on the Children's Depression Inventory: Evidence for invariance from two latent variable models. Educational and Psychological Measurement, 68, 281-303.

Carter, M. M., Miller, O., Sbrocco, T., Suchday, S., \& Lewis, E. L. (1999). Factor structure of the Anxiety Sensitivity Index among African American college students. Psychological Assessment, 11, 525-533.

Chorpita, B. F., \& Daleiden, E. L. (2000). Properties of the Childhood Anxiety Sensitivity Index in children with anxiety disorders: Autonomic and nonautonomic factors. Behavior Therapy, 31, 327-349. 
Chorpita, B. F., Albano, A. M., \& Barlow, D. H. (1996). Child anxiety sensitivity: Considerations for children with anxiety disorder. Journal of Clinical Child Psychology, 25, 77-82.

Chorpita, B. F., Yim, L., Moffit, C., Umemoto, L., \& Frances, S. E. (2000). Assessment of symptoms of DSM-IV anxiety and depression in children: A revised child anxiety and depression scale. Behaviour Research and Therapy, 38, 835-855.

Clark, D. M. (1986). A cognitive approach to panic. Behaviour Research and Therapy, $24,461-470$.

Cox, B. J., Parker, J. D., \& Swinson, R. P. (1996). Anxiety sensitivity: confirmatory evidence for a multidimensional construct. Behaviour Research and Therapy, 34, $591-598$.

Craighead, W. E., Smucker, M. R., Craighead, L. W., \& Ilardi, S. S. (1998). Factor analysis of the Children's Depression Inventory in a community sample. Psychological Assessment, 10, 156-165.

Dia, D. A., \& Bradshaw, W. (2008). Cognitive risk factors to the development of anxiety and depressive disorders in adolescents. Child and Adolescent Social Work Journal, 25, 469-481.

Essau, C. A., Sasagawa, S., \& Ollendick, T. H. (2010). The facets of anxiety sensitivity in adolescents. Journal of Anxiety Disorders, 24, 23-29.

Fan, X., Thompson, B., \& Wang, L. (1999). Effects of sample size, estimation method, and model specification on structural equation modeling fit indexes. Structural Equation Modeling, 6, 56-83.

Hayward, C., Killen, J. D., Kraemer, H. C., \& Taylor, B. (2000). Predictors of panic attacks in adolescents. Journal of the American Academy of Child and Adolescent Psychiatry, 39, 207-214.

Honaker, J., King, G., \& Blackwell, M. (2010). Amelia II: Program for Missing Data (Version 1.2-17) [Computer Software]. Retrieved from http://gking.harvard.edu/

Hu, L., \& Bentler, P. M. (1999). Cutoff criteria for fit indexes in covariance structure analysis: Conventional criteria versus new alternatives. Structural Equation Modeling, 6, 1-55.

Hui, C. H., \& Triandis, H. C. (1985). Measurement in cross-cultural psychology: A review and comparison of strategies. Journal of Cross-Cultural Psychology, 16, $131-152$. 
Ialongo, N. S., Kellam, S. G., \& Poduska, J. (1999). Manual for the Baltimore How I Feel (Tech. Rep. No. 2). Baltimore: John Hopkins University.

Jaccard, J., \& Wan, C. H. (1996). LISREL approaches to interaction effects in multiple regression. Thousand Oaks, CA: Sage Publications.

Joiner, T. E., Schmidt, N. B., Schmidt, K. L., Laurent, J., Catanzaro, S. J., Perez, M., et al. (2002). Anxiety sensitivity as a specific and unique marker of anxious symptoms in youth psychiatric inpatients. Journal of Abnormal Child Psychology, $30,167-175$.

Kearney, C. A., Albano, A. M., Eisen, A. R., Allan, W. D., \& Barlow, D. H. (1997). The phenomenology of panic disorders in youngsters: an empirical study of a clinical sample. Journal of Anxiety Disorders, 11, 49-62.

Knight, G. P., \& Zerr, A. Z. (2010). Introduction to the Special Section: Measurement Equivalence in Child Development Research. Child Development Perspectives, $4,1-4$.

Kovacs, M. (1992). Children's Depression Inventory Manual. North Tonawanda, NY: Multi-Health Systems, Inc.

Lambert, S. F., Cooley, M., Campbell, K. D., Benoit, M. Z., \& Stansbury, R. (2004). Assessing anxiety sensitivity in inner-city African American children: Psychometric properties of the Child Anxiety Sensitivity Index. Journal of Clinical Child and Adolescent Psychology, 33, 248-259.

Lambert, S. F., McCreary, B. T., Preston, J. L., Schmidt, N. B., Joiner, T. E., \& Ialongo, N. S. (2004). Anxiety sensitivity in African-American adolescents: evidence of symptom specificity of anxiety sensitivity components. Journal of the American Academy of Child and Adolescent Psychiatry, 43, 887-895.

Lau, J. J., Calamari, J. E., \& Waraczynski, M. (1996). Panic attack symptomatology and anxiety sensitivity in adolescents. Journal of Anxiety Disorders, 10, 355-364.

Laurent, J., Catanzaro, S., Joiner, T., Rudolph, K., Potter, K., Lambert, S., et al. (1999). A measure of positive and negative affect for children: Scale development and preliminary validation. Psychological Assessment, 11, 326-338.

Laurent, J., Schmidt, N. B., Catanzaro, S., Joiner, T. E., \& Kelly, A. (1998). Anxiety sensitivity in children: Evidence for unidimensionality. Journal of Anxiety Disorders, 12, 307-331. 
Lilienfeld, S. O., Jacob, R. G., \& Turner, S. M. (1989). Comment on Holloway and McNally's (1987) "Effects of anxiety sensitivity on the response to hyperventilation". Journal of Abnormal Psychology, 98, 100-102.

Marian, E., \& McNally, R. J. (1996). Anxiety sensitivity, suffocation, fear, trait anxiety, and breathholding. Behaviour Research and Therapy, 34, 603-607.

McLaughlin, K. M., \& Hatzenbuehler, M. L. (2009). Stressful life events, anxiety sensitivity and internalizing symptoms in adolescents. Journal of Abnormal Psychology, 118, 659-669.

McNally, R. J. (1996). Anxiety sensitivity is distinguishable from trait anxiety. In R. M. Rapee (Ed.), Current Controversies in the Anxiety Disorders (pp. 245-248). New York: Guilford Press.

McNally, R. J. (1989). Is anxiety sensitivity distinguishable from trait anxiety: a reply to Lilienfeld, Jacob and Turner? Journal of Abnormal Psychology, 98, 193-94.

McNally, R. J. (1999). Theoretical approaches to the fear of anxiety. In S. Taylor (Ed.), Anxiety Sensitivity: Theory, Research and Treatment (pp. 3-16). London: Lawrence Erlbaum Associates.

Millsap, R. E., \& Yun-Tein, J. (2004). Assessing factorial invariance in orderedcategorical measures. Multivariate Behavioral Research, 39, 479-515.

Muris, P., Schmidt, H., Merckelbach, H., \& Schouten, E. (2001). Anxiety sensitivity in adolescents: factor structure and relationships to trait anxiety and symptoms of anxiety disorders and depression. Behaviour Research and Therapy, 39, 89-100.

Muthén, B. O., \& Asparouhov, T. (2002). Latent variable analysis with categorical outcomes: Multiple-group and growth modeling in Mplus. Muthén \& Muthén, Mplus Web Notes: No. 4, Version 5.

Muthén, L. K., \& Muthén, B. O. (2007). Mplus User's Guide (5th ed.). Los Angeles: Muthén \& Muthén.

Naragon-Gainey, K. (2010). Meta-analysis of the relations of anxiety sensitivity to the depressive and anxiety disorders. Psychological Bulletin, 136, 128-150.

Norton, R. G., Dorward, J., \& Cox, B. J. (1986). Factors associated with panic attacks on nonclinical subjects. Behavior Therapy, 17, 239-252.

Olatjuni, B. O., \& Wolitzky-Taylor, K. B. (2009). Anxiety sensitivity and the anxiety disorders: a meta-analytic review and synthesis. Psychological Bulletin, 135, 974999. 
Ollendick, T. H. (1983). Reliability and validity of the revised Fear Survey Schedule for Children (FSSC-R). Behavior Research and Therapy, 21, 685-692.

Otto, M. W., Pollack, M. H., Fava, H. M., Ucello, R., \& Rosenbaum, J. F. (1995). Elevated anxiety sensitivity index scores in patients with major depression: correlates and changes with antidepressant treatment. Journal of Anxiety Disorders, 9, 117-123.

Pela, O. A., \& Reynolds, C. R. (1982). Cross-cultural application of the Revised Children's Manifest Anxiety Scale: Normative and reliability data for Nigerian primary school children. Psychological Reports, 5, 1135-1138.

Peterson, R. A., \& Reiss, S. (1987). Anxiety Sensitivity Index Manual. Orland Park, IL: International Diagnostic Systems.

Pina, A. A., \& Silverman, W. K. (2004). Clinical phenomenology, somatic symptoms, and distress in Hispanic/Latino and European/American youths with anxiety disorders. Journal of Clinical Child and Adolescent Psychology, 33, 227-236.

Pina, A. A., Little, M., Knight, G. P., \& Silverman, W. K. (2009). Cross-ethnic measurement equivalence of the RCMAS in Latino and White youth with anxiety disorders. Journal of Personality Assessment, 91, 58-61.

Rabian, B., Peterson, R. A., Richters, J., \& Jensen, P. S. (1993). Anxiety sensitivity among anxious children. Journal of Clinical Child Psychology, 22, 441-446.

Reiss, S. (1991). Expectancy model of fear, anxiety and panic. Clinical Psychology Review, 11, 141-153.

Reiss, S. (1987). Theoretical perspectives on the fear of anxiety. Clinical Psychology Review, 7, 585-596.

Reiss, S. (1997). Trait anxiety: it's not what you think it is. Journal of Anxiety Disorders, 11, 201-214.

Reiss, S., \& McNally, R. J. (1985). The expectancy model of fear. In S. Reiss, \& R. R. Bootzin (Eds.), Theoretical Issues in Behavior Therapy (pp. 107-121). New York: Academic Press.

Reiss, S., Peterson, R. A., Gursky, D. M., \& McNally, R. J. (1986). Anxiety sensitivity, anxiety frequency and the predictions of fearfulness. Behaviour Research and Therapy, 24, 1-8. 
Reiss, S., Silverman, W. K., \& Weems, C. F. (2001). Anxiety sensitivity. In M. W. Vasey, \& M. R. Dadds (Eds.), The Developmental Psychopathology of Anxiety (pp. 92-111). New York: Oxford University Press.

Reynolds, C. R., \& Richmond, B. O. (1985). Revised Children's Manifest Anxiety Scale: manual. Los Angeles: Western Psychological Services.

Reynolds, C. R., \& Richmond, B. O. (1978). What I think and feel: A revised measure of children's manifest anxiety. Journal of Abnormal Child Psychology, 6, 271-280.

Schmidt, N. B., Lerew, D. R., \& Joiner, T. E. (1998). The role of anxiety sensitivity in the pathogenesis of anxiety and depression: Evidence for symptom specificity. Behaviour Research and Therapy, 36, 165-177.

Schumacker, R. E., \& Lomax, R. G. (2004). A Beginner's Guide to Structural Equation Modeling (2nd ed.). Manwah, NJ: Lawrence Erlbaum Associates.

Silverman, W. K., \& Albano, A. M. (1996). Anxiety Disorders Interview Schedule for Children. San Antonio, TX: Psychological Corporation.

Silverman, W. K., \& Nelles, W. B. (1988). The Anxiety Disorders Interview Schedule for Children. Journal of the American Academy of Child and Adolescent Psychiatry, 27, 772-778.

Silverman, W. K., \& Weems, C. F. (1999). Anxiety sensitivity in children. In S. Taylor (Ed.), Anxiety sensitivity: Theory, research and the treatment of the fear of anxiety (pp. 239-268). London: Lawrence Erlbaum Associates.

Silverman, W. K., Fleisig, W., Rabian, B., \& Peterson, R. A. (1991). Childhood Anxiety Sensitivity. Journal of Clinical Child Psychology, 20, 162-168.

Silverman, W. K., Ginsburg, G. S., \& Goedhart, A. W. (1999). Factor structure of the Childhood Anxiety Sensitivity Index. Behaviour Research and Therapy, 37, 903917.

Silverman, W. K., Goedhart, A. W., Barret, P., \& Turner, C. (2003). The facets of anxiety sensitivity represented in the childhood anxiety sensitivity index: Confirmatory analyses of factor models from past studies. Journal of Abnormal Psychology, $112,364-374$.

Silverman, W. K., Saavedra, L. M., \& Pina, A. A. (2001). Test-retest reliability of the anxiety symptoms and diagnoses with the Anxiety Disorders Interview Schedule for DSM-IV: Child and parent versions. Journal of the American Academy of Child and Adolescent Psychiatry, 40, 937-944. 
Spence, S. H. (1998). A measure of anxiety symptoms among children. Behaviour Research and Therapy, 36, 545-566.

Spielberger, C. D. (1973). Manual for the State-Trait Anxiety Inventory for Children. Palo Alto, CA: Consulting Psychologists Press.

Stewart, S. H., Samoluk, S. B., \& MacDonald, A. B. (1999). Anxiety sensitivity and substance use and abuse. In S. Taylor (Ed.), Anxiety Sensitivity: Theory, Research and Treatment of the Fear of Anxiety (pp. 287-320). London: Lawrence Erlbaum Associates.

Taylor, S. (1995). Anxiety sensitivity: theoretical perspectives and recent findings. Behaviour Research and Therapy, 33, 243-258.

Taylor, S., \& Fedoroff, I. C. (1999). The expectancy theory of fear, anxiety and panic: a conceptual and empirical analysis. In S. Taylor (Ed.), Anxiety Sensitivity: Theory, Research and Treatment of the Fear of Anxiety (pp. 17-34). London: Lawrence Erlbaum Associates.

Taylor, S., Koch, W. J., \& Mcnally, R. J. (1992). How does anxiety sensitivity vary across the anxiety disorders? Journal of Anxiety Disorders, 6, 249-259.

Taylor, S., Koch, W. J., McNally, R. J., \& Crockett, D. J. (1992). Conceptualizations of Anxiety Sensitivity. Psychological Assessment, 4, 245-250.

Taylor, S., Koch, W. J., Woody, S., \& McLean, P. (1996). Anxiety sensitivity and depression: How are they related? Journal of Abnormal Psychology, 105, 474479 .

U.S. Census Bureau. (2010). 2009 National Characteristics Estimates. Retrieved from U.S. Census Bureau website: http://www.census.gov/popest/estimates.html.

Vandenberg, R. J., \& Lance, C. E. (2000). A review and synthesis of the measurement invariance literature: Suggestions, practices and recommendations for organizational research. Organizational Research Methods, 3, 4-70.

Varela, R. E., \& Hensley-Maloney, L. (2009). The influence of culture on anxiety in Latino youth: A review. Clinical Child and Family Psychology Review, 12, 217233.

Varela, R. E., Weems, C. F., Berman, S. L., Hensley, L., \& Rodriguez de Bernal, M. C. (2007). Internalizing symptoms in Latinos: The role of anxiety sensitivity. Journal of Youth Adolescence, 36, 429-440. 
Vasey, M. W., \& Dadds, M. (Eds.). (2001). The Developmental Psychopathology of Anxiety. New York: Oxford University Press.

Weems, C. F., Hammond-Laurence, K., Silverman, W. K., \& Ferguson, C. (1997). The relation between anxiety sensitivity and depression in children and adolescents referred for anxiety. Behaviour Research and Therapy, 35, 961-966.

Weems, C. F., Hammond-Laurence, K., Silverman, W. K., \& Ginsburg, G. (1998). Testing the utility of the anxiety sensitivity construct in children and adolescents referred for anxiety disorders. Journal of Clinical Child Psychology, 24, 69-77.

Weems, C. F., Hayward, C., Killen, J., \& Taylor, C. B. (2002). A longitudinal investigation of anxiety sensitivity in adolescence. Journal of Abnormal Psychology, 111, 471-477.

Widaman, K. F., Ferrer, E., \& Conger, R. D. (2010). Factorial invariance within longitudinal structural equation models: Measuring the same construct across time. Child Development Perspectives, 4, 10-18. 
VITA

\section{KRISTIN NICHOLS-LOPEZ}

February 25, 1981

2002

2006

$2005-2010$
Born, Miami, Florida

B.A., Psychology

Emory University

Atlanta, Georgia

M.S., Psychology

Florida International University

Miami, Florida

Doctoral Candidate in Psychology

Florida International University

Miami, Florida

Director, Assessment Research and Data Analysis

Miami-Dade County Public Schools

Office of Program Evaluation

Miami, Florida

\section{PUBLICATIONS AND PRESENTATIONS}

Franco, X., Saavedra, L. M., Nichols-Lopez, K. A. \& Silverman, W. K. Identification of childhood behavior problems via mother/child interactions. Poster presented at the Biennial Meeting for the Society for Research in Child Development. Boston, MA. March 2007.

Saavedra, L. M., Franco, X., Nichols-Lopez, K. A. \& Silverman, W. K. Children who received treatment for internalizing behavior problems grow-up: An 8-to-13 year follow up. Poster presented at the Biennial Meeting for the Society for Research in Child Development. Boston, MA. March 2007.

Marin, C. E., Rey, Y., Nichols-Lopez, K. A., \& Silverman, W. K. Parenting practices as related to child perception of control in the development childhood anxiety disorders. Poster presented at the Annual Meeting for the American Psychological Association. San Francisco, CA. August 2007.

Muñiz-Leen, A. M., Nichols-Lopez, K. A., Marin, C. E., Rey, Y., Hernandez, M., \& Silverman, W. K. Anxiety sensitivity's facets in relation to anxious and depressive symptoms. Poster presented at the Annual Meeting for the American Academy of Child and Adolescent Psychiatry. Boston, MA, October 2007 
Urdegar, S.M., Arcia, E., Nichols-Lopez, K. A. (2007). Title I Evaluation Summary Report, 2005-06. Miami, FL: Miami-Dade County Public Schools.

Nichols-Lopez, K. A. (2008). Limited English Proficient Students and their Academic Progress: 2006-07. Miami, FL: Miami-Dade County Public Schools.

Nichols-Lopez, K. A. (2008). 2007 Academic Achievement of Haitian English Language Learners. Miami, FL: Miami-Dade County Public Schools.

Marin, C. E., Rey, Y., Nichols-Lopez, K. A., \& Silverman, W. K. (2008). The relations between anxiety sensitivity and anxiety control in the prediction of anxiety symptoms among children and adolescents. Behavioural and Cognitive Psychotherapy, 36, 391-402. 